\title{
Searching for chemical signatures of brown dwarf formation ${ }^{\star}$
}

\author{
J. Maldonado ${ }^{1}$ and E. Villaver ${ }^{2}$
}

\author{
1 INAF-Osservatorio Astronomico di Palermo, Piazza del Parlamento 1, 90134 Palermo, Italy \\ e-mail: jmaldonado@astropa.inaf.it \\ 2 Universidad Autónoma de Madrid, Dpto. Física Teórica, Módulo 15, Facultad de Ciencias, Campus de Cantoblanco, 28049 Madrid, \\ Spain
}

Received 22 November 2016 / Accepted 9 February 2017

\begin{abstract}
Context. Recent studies have shown that close-in brown dwarfs in the mass range 35-55 $M_{\text {Jup }}$ are almost depleted as companions to stars, suggesting that objects with masses above and below this gap might have different formation mechanisms.

Aims. We aim to test whether stars harbouring massive brown dwarfs and stars with low-mass brown dwarfs show any chemical peculiarity that could be related to different formation processes.

Methods. Our methodology is based on the analysis of high-resolution échelle spectra $(R \sim 57000)$ from $2-3 \mathrm{~m}$ class telescopes. We determine the fundamental stellar parameters, as well as individual abundances of $\mathrm{C}, \mathrm{O}, \mathrm{Na}, \mathrm{Mg}, \mathrm{Al}, \mathrm{Si}, \mathrm{S}, \mathrm{Ca}, \mathrm{Sc}, \mathrm{Ti}, \mathrm{V}, \mathrm{Cr}$, $\mathrm{Mn}, \mathrm{Co}, \mathrm{Ni}$, and $\mathrm{Zn}$ for a large sample of stars known to have a substellar companion in the brown dwarf regime. The sample is divided into stars hosting massive and low-mass brown dwarfs. Following previous works, a threshold of $42.5 M_{\text {Jup }}$ was considered. The metallicity and abundance trends of the two subsamples are compared and set in the context of current models of planetary and brown dwarf formation.

Results. Our results confirm that stars with brown dwarf companions do not follow the well-established gas-giant planet metallicity correlation seen in main-sequence planet hosts. Stars harbouring massive brown dwarfs show similar metallicity and abundance distribution as stars without known planets or with low-mass planets. We find a tendency of stars harbouring less-massive brown dwarfs of having slightly higher metallicity, $\left[\mathrm{X}_{\mathrm{Fe}} / \mathrm{Fe}\right]$ values, and abundances of Sc II, Mn I, and Ni I than the stars having the massive brown dwarfs. The data suggest, as previously reported, that massive and low-mass brown dwarfs might present differences in period and eccentricity.

Conclusions. We find evidence of a non-metallicity dependent mechanism for the formation of massive brown dwarfs. Our results agree with a scenario in which massive brown dwarfs are formed as stars. At high metallicities, the core-accretion mechanism might become efficient in the formation of low-mass brown dwarfs, while at lower metallicities low-mass brown dwarfs could form by gravitational instability in turbulent protostellar discs.
\end{abstract}

Key words. techniques: spectroscopic - stars: abundances - stars: late-type - planetary systems

\section{Introduction}

Understanding whether brown dwarfs and giant planets share similar formation mechanisms is the subject of intensive studies (e.g. Luhman et al. 2007; Whitworth et al. 2007; Burgasser 2011; Luhman 2012; Chabrier et al. 2014).

The standard definition of a brown dwarf includes objects in a wide range of masses, from 13 to 80 Jupiter masses, with sufficient mass to ignite deuterium but below the hydrogen-burning minimum mass (Burrows et al. 1997, 2001; Chabrier \& Baraffe 2000; Spiegel et al. 2011). It is now well established that there is a paucity of close brown dwarf companions in comparison with gas-giant planets or binaries around main-sequence stars

\footnotetext{
* Based on observations made with the Mercator Telescope; on observations made with the Nordic Optical Telescope; on data products from the SOPHIE archive; on data products from the ELODIE archive; and on data products from observations made with ESO Telescopes at the La Silla Paranal Observatory under programmes ID 072. C-0488(E), 076.C-0155(A), 076.C-0429(A), 078.C-0133(A), 079.C-0329(A), 082.C-0333(A), 083.C-0174(A), 083.C-0413(A), 085. C-0019(A), 085.C-0393(A), 087.A-9029(A), 087.C-0831(A), 090.C0421(A), 093.C-0409(A), 094.D-0596(A), 095.A-9029(C), 178.D0361(B), 183.C-0972(A), 184.C-0639(A), and 188.C-0779(A)
}

(Campbell et al. 1988; Murdoch et al. 1993; Marcy \& Butler 2000; Grether \& Lineweaver 2006; Sahlmann et al. 2011), usually known as the brown dwarf desert.

There have been several studies with the goal of understanding whether the properties of the brown dwarf population could be related to the formation mechanism of these objects. In a recent work, Ma \& Ge (2014) compare the orbital properties (period and eccentricities) of a sample of brown dwarf companions around 65 stars. They found that while brown dwarfs with minimum masses greater than $\sim 42.5 M_{\text {Jup }}$ follow a similar periodeccentricity distribution to that of stellar binaries, brown dwarfs with masses below $42.5 M_{\text {Jup }}$ have an eccentricity distribution consistent with that of massive planets. This suggests that the standard definition of a brown dwarf might mix two kinds of objects with different formation mechanisms. The formation of high-mass brown dwarfs might be a scaled-down version of star formation through fragmentation of molecular clumps. On the other hand, less-massive brown dwarfs might form as giant planets do.

Current models of giant-planet formation can be divided into two broad categories: i) core-accretion models (e.g. Pollack et al. 1996; Rice \& Armitage 2003; Alibert et al. 2004; Mordasini et al. 2012) which are able to explain the observed 
gas-giant planet metallicity correlation (e.g. Gonzalez 1997; Santos et al. 2004; Fischer \& Valenti 2005) as well as the lack of a metallicity correlation in low-mass planet hosts (e.g. Mayor et al. 2011; Cassan et al. 2012; Howard et al. 2013) and ii) disc instability models which do not depend on the metallicity of the primordial disc (Boss 1997, 2002, 2006). If brown dwarfs form in the same way as giant-planets and those are mainly formed by core-accretion, stars hosting brown dwarfs should show the metal-enrichment seen in gas-giant planet hosts.

Several attempts to understand the metallicity distribution of stars with brown dwarf companions have been performed. Sahlmann et al. (2011) notes that while some stars with brown dwarf companions are metal rich, others show subsolar metallicities. Ma \& Ge (2014) do not find significant metallicity differences between brown dwarf host stars with (minimum) masses below and above $42.5 M_{\text {Jup }}$. Mata Sánchez et al. (2014) analyse in a homogeneous way the abundances of 15 stars hosting brown dwarfs ( 7 candidates and 8 discarded based on their HIPPARCOS astrometry) showing that they differ from those of stars hosting gas-giant planets. Also, they suggest higher abundances for the stars hosting brown dwarfs with masses below $42.5 M_{\text {Jup }}$.

Given that previous works are based on small or inhomogeneous samples a detailed chemical analysis of a homogeneous and large sample of stars hosting brown dwarfs is needed before formation mechanisms of brown dwarfs are invoked. This is the goal of this paper, in which we present a homogeneous analysis of a large sample of brown dwarf hosts that is based on highresolution and high signal-to-noise ratio $(\mathrm{S} / \mathrm{N})$ échelle spectra.

This paper is organised as follows. Section 2 describes the stellar samples analysed in this work, the spectroscopic observations, and how stellar parameters and abundances are obtained. The comparison of the properties and abundances of stars with brown dwarf companions with masses above and below 42.5 $M_{\text {Jup }}$ is presented in Sect. 3, where we also include a search for correlations between the stellar and brown dwarf properties. The results are discussed at length in Sect. 4. Our conclusions follow in Sect. 5.

\section{Observations}

\subsection{Stellar sample}

A sample of stars with known brown dwarfs companions (SWBDs) with (projected) masses between 10 and $70 M_{\text {Jup }}$ was built using as reference the 65 stars listed in the recent compilation by Ma \& Ge (2014) plus the 61 stars with brown dwarf candidates listed by Wilson et al. (2016). Although different authors might have different criteria for classifying an object as a brown dwarf, we note that $\sim 64 \%$ of the stars listed in Wilson et al. (2016) were already given in the compilation by $\mathrm{Ma} \& \mathrm{Ge}$ (2014). Fifteen brown dwarf companions listed in Wilson et al. (2016) were published after Ma \& Ge (2014). Only seven stars listed in Wilson et al. (2016) and known before Ma \& Ge (2014) were not included in this compilation: five of them have projected masses $\sim 10-11 M_{\text {Jup }}$, while the other two are in the range $62-65 M_{\text {Jup }}$. Although we do not know the reason why these seven stars were not included in Ma \& Ge (2014), we have decided to keep them in our analysis. Thus, from the above compilations, we selected all stars with spectral types between F6 and K2 (independently of the luminosity class) with high-resolution spectra available in public archives or already observed by our team in our previous programmes (see below). Several stars having a very low $\mathrm{S} / \mathrm{N}$ or showing indications of high rotation were also discarded.
Table 1. Properties of the different spectrographs used in this work.

\begin{tabular}{lccc}
\hline \hline Spectrograph & Spectral range $(\AA)$ & Resolving power & $N$ stars \\
\hline SOPHIE & $3872-6943$ & 75000 & 20 \\
HARPS & $3780-6910$ & 115000 & 16 \\
ELODIE & $3850-6800$ & 42000 & 6 \\
FIES & $3640-7360$ & 67000 & 4 \\
FEROS & $3500-9200$ & 48000 & 3 \\
HERMES & $3800-9000$ & 85000 & 2 \\
HARPS-N & $3830-6930$ & 115000 & 1 \\
UVES & $4780-6800$ & 110000 & 1 \\
\hline
\end{tabular}

Our final sample consists of 53 stars with brown dwarfs, including $10 \mathrm{~F}$ stars, $31 \mathrm{G}$ stars, and $12 \mathrm{~K}$ stars. Regarding their evolutionary stage, 8 stars are red giants, 19 are classified as subgiants, while 26 stars are on the main sequence. The stars are listed in Table A.1.

\subsection{Spectroscopic data}

High-resolution spectra of the stars were mainly collected from public archives: data for 6 stars were taken from the ELODIE (Baranne et al. 1996) archive $^{1}, 20$ stars from the SOPHIE (Bouchy \& Sophie Team 2006) archive ${ }^{2}$, HARPS (Mayor et al. 2003) spectra from the ESO archive $^{3}$ was used for 16 stars, while for 3 stars FEROS spectra were used (Kaufer et al. 1999). A UVES (Dekker et al. 2000) spectra was taken from the ESO archive for the star 11 Com, and HARPS-N spectra (Cosentino et al. 2012) were taken from the Telescopio Nazionale Galileo archive ${ }^{4}$ for the star KOI-415. Additional data for six stars were taken from our own observations (Maldonado et al. 2013; Maldonado \& Villaver 2016), four of them using the Nordic Optical Telescope $(2.56 \mathrm{~m})$ with the FIES instrument (Frandsen \& Lindberg 1999), and two using the Mercator telescope $(1.2 \mathrm{~m})$ with the HERMES spectrograph (Raskin et al. 2011). Table 1 summarises the properties of the different spectra.

All the spectra were reduced by the corresponding pipelines which implement the typical corrections involved in échelle spectra reduction. When needed several spectra of the same star were properly combined in order to obtain higher $\mathrm{S} / \mathrm{N}$ spectra. Typical values of the $\mathrm{S} / \mathrm{N}$ (measured around $605 \mathrm{~nm}$ ) are between 70 and 200. The spectra were corrected from radial velocity shifts by using the precise radial velocities provided by the ELODIE, SOPHIE, and HARPS data reduction pipelines. For the rest of the targets radial velocities were measured by cross-correlating their spectra with spectra of radial velocity standard stars of similar spectral types obtained during the same observations.

\subsection{Analysis}

Basic stellar parameters $\left(T_{\mathrm{eff}}, \log g\right.$, microturbulent velocity $\xi_{\mathrm{t}}$, and $[\mathrm{Fe} / \mathrm{H}]$ ) were determined by applying the iron ionisation and excitation equilibrium conditions to a set of well-defined $302 \mathrm{Fe}$ I and $28 \mathrm{Fe}$ II lines. The computations were done with the TGVIT (Takeda et al. 2005) code. The line list and details

\footnotetext{
1 http://atlas.obs-hp.fr/elodie/

2 http://atlas.obs-hp.fr/sophie/

http://archive.eso.org/wdb/wdb/adp/phase3_spectral/

form?phase3_collection=HARPS

4 http://ia2.oats.inaf.it/archives/tng
} 

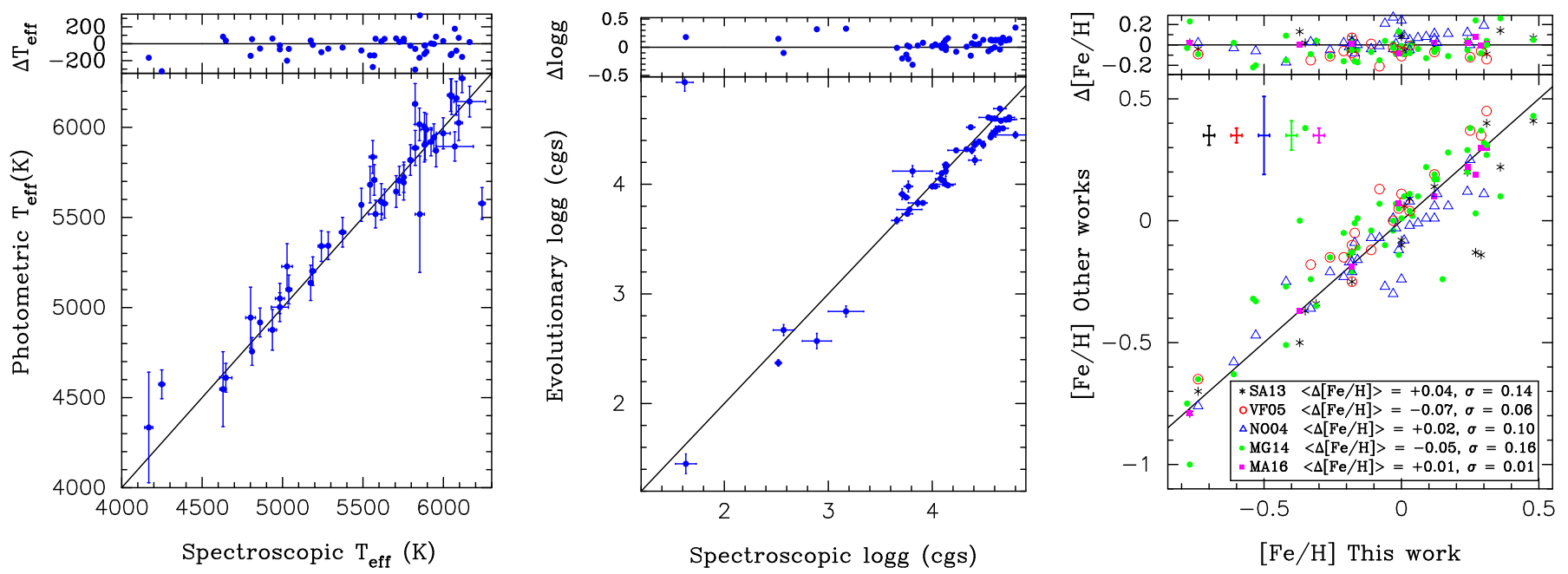

Fig. 1. Spectroscopic stellar parameters derived in this work vs. estimates based on photometry or values from the literature. Left: effective temperature; middle: surface gravity $\log g$; right: stellar metallicity $[\mathrm{Fe} / \mathrm{H}]$. The upper parts show the differences between the parameters derived in this work and the values obtained from photometry or given in the literature.

on the adopted parameters (excitation potential, $\log (g f)$ values) are available on Y. Takeda's web page ${ }^{5}$. ATLAS9, planeparallel, local thermo-dynamic equilibrium (LTE) atmosphere models (Kurucz 1993) were used in the computations. Uncertainties in the stellar parameters are obtained by changing each stellar parameter from the converged solution until the excitation equilibrium, the ionisation equilibrium, or the match of the curve of growth is no longer fulfilled. We are aware that this procedure only evaluates statistical errors and that other systematic sources of uncertainties (i.e. the line lists used, the adopted atomic parameters, or the choice of the atmosphere model) are not taken into account (for details see Takeda et al. 2002a,b). Zieliński et al. (2012) estimated that more realistic uncertainties might be of the order of two to three times those provided by TGVIT.

In order to avoid weak lines, as well as errors due to uncertainties in the damping parameters, only lines with measured equivalent widths (EWs) between 8 and $120 \mathrm{m \AA}$ were used (e.g. Takeda et al. 2008). Stellar EWs were measured using the automatic code ARES2 (Sousa et al. 2015) adjusting the reject parameter according to the $\mathrm{S} / \mathrm{N}$ ratio of the spectra as described in Sousa et al. (2008).

\subsection{Photometric parameters and comparison with previous works}

In order to test the reliability of our derived parameters, photometric effective temperatures were derived from the HIPPARCos $(B-V)$ colours (Perryman \& ESA 1997) by using the calibration provided by Casagrande et al. (2010, Table 4). Before computation, colours were de-reddened by using the stellar galactic coordinates and the tables given by Arenou et al. (1992). Distances were obtained from the revised parallaxes provided by van Leeuwen (2007) from a new reduction of the HIPPARCos raw data. In the few cases where colours or parallaxes were not available we took the values provided by the Simbad database (Wenger et al. 2000). The comparison between the temperature values obtained by spectroscopic and by photometric procedures is shown in the left panel of Fig. 1. It is clear from the figure that there is no clear systematic difference between them. The

\footnotetext{
5 http://optik2.mtk.nao.ac.jp/ takeda/tgv/
}

temperatures differ in median by $21 \mathrm{~K}$, with a standard deviation of $153 \mathrm{~K}$.

Stellar evolutionary parameters, namely surface gravity, age, mass, and radius, were computed from HIPPARCOS $V$ magnitudes and parallaxes using the code PARAM ${ }^{6}$ (da Silva et al. 2006), which is based on the use of Bayesian methods, and the parsec set of isochrones by Bressan et al. (2012). The comparison between the spectroscopic and evolutionary $\log g$ values is shown in the middle panel of Fig. 1. The figure reveals the known trend of spectroscopic surface gravities to be systematically larger than the evolutionary estimates (e.g. da Silva et al. 2006; Maldonado et al. 2013). However, the distribution of $\log g_{\mathrm{spec}}-$ $\log g_{\text {evol }}$ shows a median value of only 0.05 , and a standard deviation of 0.13 consistent with previous works (da Silva et al. 2006; Maldonado et al. 2013, 2015). The outlier in the upper left corner is BD+20 2457, which has a largely undetermined parallax, $\pi=5.0 \pm 26.0$ mas (Niedzielski et al. 2009).

We finally compare our metallicities with those already reported in the literature. Values for the comparison are taken from i) the SWEETCat catalogue (Santos et al. 2013, SA13), whose parameters are mainly derived from the same authors using the iron ionisation and equilibrium conditions; ii) Valenti \& Fischer (2005, hereafter VF05), where metallicities are computed by using spectral synthesis; iii) Nordström et al. (2004, hereafter NO04), which provides photometric metallicities; iv) the compilations by Ma \& Ge (2014, hereafter MG14) and Wilson et al. (2016); and from v) Maldonado \& Villaver (2016, hereafter MA16) as a consistency double check. The comparison is shown in the right panel of Fig. 1. Overall, the agreement is good and no systematic differences are found with the literature estimates.

\subsection{Abundance computation}

Chemical abundances of individual elements $\mathrm{C}, \mathrm{O}, \mathrm{Na}, \mathrm{Mg}, \mathrm{Al}$, $\mathrm{Si}, \mathrm{S}, \mathrm{Ca}, \mathrm{Sc}, \mathrm{Ti}, \mathrm{V}, \mathrm{Cr}, \mathrm{Mn}, \mathrm{Co}, \mathrm{Ni}$, and $\mathrm{Zn}$ were obtained using the 2014 version of the code MOOG ${ }^{7}$ (Sneden 1973). The selected lines are from the list provided by Maldonado et al. (2015); the only exception is carbon, for which we use the lines

\footnotetext{
6 http://stev.oapd.inaf.it/cgi-bin/param

7 http://wWw.as.utexas.edu/ chris/moog.html
} 
Table 2. Abundance sensitivities.

\begin{tabular}{|c|c|c|c|c|c|c|c|c|c|c|c|c|}
\hline \multirow{2}{*}{ Ion } & \multicolumn{4}{|c|}{ HD 180314} & \multicolumn{4}{|c|}{ HD 38529} & \multicolumn{4}{|c|}{ HD 191760} \\
\hline & $\begin{array}{r}\Delta T_{\text {eff }} \\
\pm 53 \\
(\mathrm{~K}) \\
\end{array}$ & $\begin{array}{r}\Delta \log g \\
\pm 0.17 \\
\left(\mathrm{~cm} \mathrm{~s}^{-2}\right) \\
\end{array}$ & $\begin{array}{r}\Delta[\mathrm{Fe} / \mathrm{H}] \\
\pm 0.07 \\
(\mathrm{dex})\end{array}$ & $\begin{array}{r}\Delta \xi_{t} \\
\pm 0.21 \\
\left(\mathrm{~km} \mathrm{~s}^{-1}\right) \\
\end{array}$ & $\begin{array}{r}\Delta T_{\text {eff }} \\
\pm 43 \\
(\mathrm{~K}) \\
\end{array}$ & $\begin{array}{r}\Delta \log g \\
\pm 0.12 \\
\left(\mathrm{~cm} \mathrm{~s}^{-2}\right) \\
\end{array}$ & $\begin{array}{r}\Delta[\mathrm{Fe} / \mathrm{H}] \\
\pm 0.04 \\
(\mathrm{dex})\end{array}$ & $\begin{array}{r}\Delta \xi_{t} \\
\pm 0.14 \\
\left(\mathrm{~km} \mathrm{~s}^{-1}\right) \\
\end{array}$ & $\begin{array}{r}\Delta T_{\text {eff }} \\
\pm 10 \\
(\mathrm{~K}) \\
\end{array}$ & $\begin{array}{r}\Delta \log g \\
\pm 0.02 \\
\left(\mathrm{~cm} \mathrm{~s}^{-2}\right) \\
\end{array}$ & $\begin{array}{r}\Delta[\mathrm{Fe} / \mathrm{H}] \\
\pm 0.01 \\
(\mathrm{dex})\end{array}$ & $\begin{array}{r}\Delta \xi_{t} \\
\pm 0.05 \\
\left(\mathrm{~km} \mathrm{~s}^{-1}\right) \\
\end{array}$ \\
\hline C I & 0.06 & 0.07 & $<0.01$ & 0.01 & 0.03 & 0.04 & $<0.01$ & 0.01 & 0.01 & 0.01 & $<0.01$ & $<0.01$ \\
\hline O I & 0.01 & 0.07 & 0.03 & 0.02 & $<0.01$ & 0.06 & 0.01 & 0.02 & $<0.01$ & 0.01 & $<0.01$ & $<0.01$ \\
\hline $\mathrm{Na} \mathrm{I}$ & 0.04 & 0.02 & $<0.01$ & 0.08 & 0.02 & 0.01 & $<0.01$ & 0.02 & 0.01 & $<0.01$ & $<0.01$ & 0.01 \\
\hline Mg I & 0.03 & 0.02 & 0.01 & 0.08 & 0.03 & 0.02 & $<0.01$ & 0.04 & 0.01 & $<0.01$ & $<0.01$ & 0.01 \\
\hline $\mathrm{Al} \mathrm{I}$ & 0.03 & 0.01 & $<0.01$ & 0.05 & 0.02 & 0.01 & $<0.01$ & 0.02 & 0.01 & $<0.01$ & $<0.01$ & $<0.01$ \\
\hline Si I & 0.02 & 0.03 & 0.01 & 0.04 & $<0.01$ & 0.01 & $<0.01$ & 0.02 & $<0.01$ & $<0.01$ & $<0.01$ & 0.01 \\
\hline S I & 0.06 & 0.06 & $<0.01$ & 0.02 & 0.03 & 0.03 & $<0.01$ & 0.01 & 0.01 & $<0.01$ & $<0.01$ & $<0.01$ \\
\hline $\mathrm{Ca} \mathrm{I}$ & 0.05 & 0.03 & $<0.01$ & 0.10 & 0.03 & 0.02 & $<0.01$ & 0.05 & 0.01 & $<0.01$ & $<0.01$ & 0.01 \\
\hline Sc I & 0.07 & $<0.01$ & $<0.01$ & 0.06 & 0.04 & $<0.01$ & $<0.01$ & 0.01 & 0.01 & $<0.01$ & $<0.01$ & $<0.01$ \\
\hline Sc II & $<0.01$ & 0.07 & 0.02 & 0.10 & $<0.01$ & 0.05 & 0.01 & 0.05 & $<0.01$ & 0.01 & $<0.01$ & 0.02 \\
\hline Ti I & 0.07 & 0.01 & $<0.01$ & 0.11 & 0.05 & 0.01 & $<0.01$ & 0.04 & 0.01 & $<0.01$ & $<0.01$ & 0.01 \\
\hline Ti II & 0.01 & 0.07 & 0.02 & 0.11 & $<0.01$ & 0.05 & 0.01 & 0.06 & $<0.01$ & 0.01 & $<0.01$ & 0.02 \\
\hline V I & 0.09 & $<0.01$ & 0.01 & 0.03 & 0.05 & $<0.01$ & $<0.01$ & $<0.01$ & 0.02 & $<0.01$ & $<0.01$ & $<0.01$ \\
\hline Cr I & 0.05 & 0.01 & $<0.01$ & 0.09 & 0.03 & 0.01 & $<0.01$ & 0.04 & 0.01 & $<0.01$ & $<0.01$ & 0.01 \\
\hline Cr II & 0.03 & 0.07 & 0.02 & 0.08 & 0.01 & 0.04 & 0.01 & 0.06 & $<0.01$ & 0.01 & $<0.01$ & 0.02 \\
\hline Mn I & 0.04 & 0.02 & 0.01 & 0.14 & 0.03 & 0.01 & $<0.01$ & 0.07 & 0.01 & $<0.01$ & $<0.01$ & 0.02 \\
\hline Co I & 0.03 & 0.04 & 0.02 & 0.01 & 0.03 & 0.01 & $<0.01$ & $<0.01$ & 0.01 & $<0.01$ & $<0.01$ & $<0.01$ \\
\hline Ni I & 0.01 & 0.03 & 0.01 & 0.08 & 0.02 & $<0.01$ & $<0.01$ & 0.04 & 0.01 & $<0.01$ & $<0.01$ & 0.01 \\
\hline $\mathrm{Zn}$ I & 0.03 & 0.05 & 0.02 & 0.11 & 0.01 & 0.02 & 0.01 & 0.06 & $<0.01$ & $<0.01$ & $<0.01$ & 0.02 \\
\hline
\end{tabular}

at 505.2 and $538.0 \mathrm{~nm}$. For $\mathrm{Zn}$ abundances, the lines at 481.05 and $636.23 \mathrm{~nm}$ are used.

Hyperfine structure (HFS) was taken into account for V I and Co I abundances. HFS corrections for Mn I were not taken into account as Maldonado et al. (2015) found slightly different abundances when considering different lines. Finally, the oxygen abundance was determined from the forbidden [O I] line at $6300 \AA$. Since this line is blended with a closer Ni I line (e.g. Allende Prieto et al. 2001), we first used the MOOG task ewfind to determine the EW of the Ni line. This EW was subtracted from the EW of the Ni I plus [O I I feature. The remaining EW was used to determine the oxygen abundance (e.g. Delgado Mena et al. 2010).

We have used three representative stars, namely HD 180314 $\left(T_{\text {eff }}=4983 \mathrm{~K}\right)$, HD $38529(5578 \mathrm{~K})$, and HD $191760(5887 \mathrm{~K})$ in order to provide an estimate on how the uncertainties in the atmospheric parameters propagate into the abundance calculation. Abundances for each of these three stars were recomputed using $T_{\text {eff }}=T_{\text {eff }}+\Delta T_{\text {eff }}, T_{\text {eff }}-\Delta T_{\text {eff }}$, and similarly for $\log g, \xi_{\mathrm{t}}$, and $[\mathrm{Fe} / \mathrm{H}]$. The results are given in Table 2.

As final uncertainties for the derived abundances, we give the quadratic sum of the uncertainties due to the propagation of the errors in the stellar parameters, plus the line-to-line scatter errors. For abundances derived from one single line, a line-toline scatter error of 0.03 dex (the median value of all the scatter errors) was assumed. Abundances with large line-to-line scatter errors were discarded. We should caution that these uncertainties should be regarded as lower limits given that abundance estimates are affected by systematics (i.e. atmosphere models or atomic data) that are difficult to account for. Our abundances are given in Table A.2. They are expressed relative to the solar values derived in Maldonado et al. (2015) and Maldonado \& Villaver (2016), which were obtained by using similar spectra to and the same methodology as the one used in this work.

A comparison of our derived abundances with those previously reported in the literature is shown in Fig. 2. While individual comparisons among this work and those in the literature are difficult to perform given the small number of stars in common and the different species analysed, there seems to be an overall good agreement between our estimates and previously reported values for the refractory elements. In the case of volatile elements $(\mathrm{C}, \mathrm{O}, \mathrm{S}$, and $\mathrm{Zn}$ ) we found few previous estimates to compare with, most likely because of the inherent difficulties in obtaining accurate abundances for these elements.

\section{Results}

\subsection{Abundance patterns of stars with brown dwarfs}

Recent studies (Sahlmann et al. 2011; Ma \& Ge 2014; Mata Sánchez et al. 2014) have suggested that the formation mechanisms of BDs companions with masses above and below $\sim 42.5 M_{\text {Jup }}$ may be different. Therefore, we have divided the sample of stars with brown dwarfs into stars with BDs candidates in the mass range $M_{\mathrm{C}} \sin i<42.5 M_{\text {Jup }}$ and stars with BDs candidates with masses $M_{\mathrm{C}} \sin i>42.5 M_{\mathrm{Jup}}{ }^{8}$.

The SWBDs with candidates in the mass range $M_{\mathrm{C}} \sin i<$ $42.5 M_{\text {Jup }}$ is composed of 32 stars, including $4 \mathrm{~F}$ stars, $19 \mathrm{G}$ stars, and $9 \mathrm{~K}$ stars. Regarding the evolutionary state of the candidates, 8 are giants, 12 are classified as subgiants, and 12 stars are on the main sequence. The total number of stars in the SWBDs with $M_{\mathrm{C}} \sin i>42.5 M_{\mathrm{Jup}}$ is 21 , including $6 \mathrm{~F}$ stars, $12 \mathrm{G}$ stars, and $3 \mathrm{~K}$ stars. The sample is composed of 14 main-sequence stars as well as 7 stars in the subgiant branch. In the following we compare the metallicities and abundances of these two subsamples.

\subsubsection{Stellar biases}

Before a comparison of the metallicities and individual abundances between the two defined samples of stars with brown dwarf candidates is made, a comparison of the stellar properties of the two samples was performed, in particular in terms of age, distance, and kinematics, since these parameters are

\footnotetext{
8 Throughout the text we use the notation "minimum mass $M_{\mathrm{C}} \sin i$ "; however, there are seven candidates with true mass determinations (four in the mass range $M_{\mathrm{C}}>42.5 M_{\mathrm{Jup}}$ and three with $\left.M_{\mathrm{C}}<42.5 M_{\mathrm{Jup}}\right)$.
} 
J. Maldonado and E. Villaver: Searching for chemical signatures of brown dwarf formation

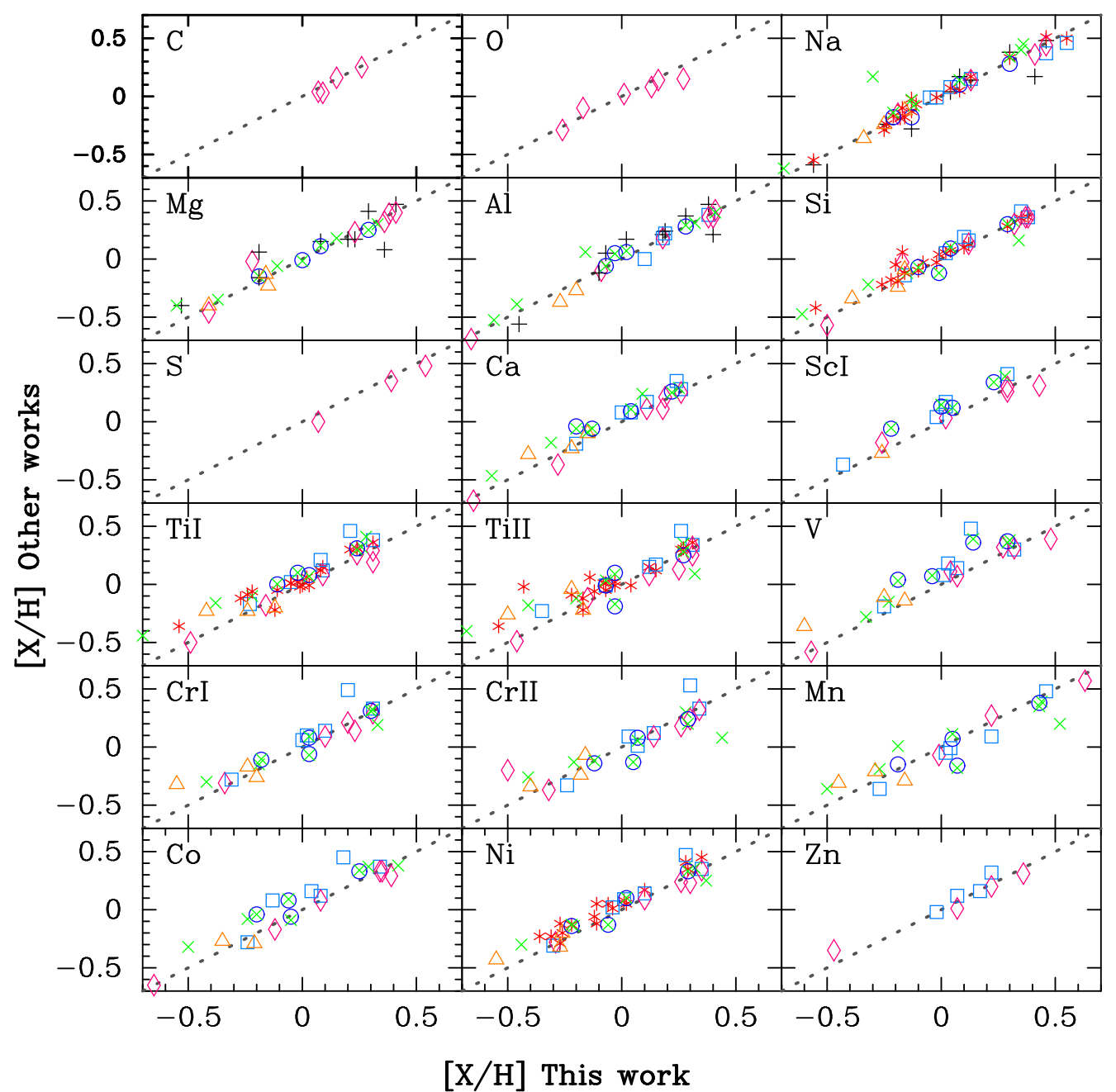

Fig. 2. Comparison of our abundances to those of Beirão et al. (2005; black +), Valenti \& Fischer (2005; red *), Takeda (2007; cyan open squares), Neves et al. (2009; blue open circles), Adibekyan et al. (2012; green X), Mata Sánchez et al. (2014; orange open triangles), and Maldonado \& Villaver (2016; purple open diamonds).

Table 3. Comparison between the properties of SWBDs with $M_{\mathrm{C}} \sin i<42.5 M_{\mathrm{Jup}}$ and SWBDs with $M_{\mathrm{C}} \sin i>42.5 M_{\mathrm{Jup}}$.

\begin{tabular}{|c|c|c|c|c|c|c|c|c|}
\hline & \multicolumn{3}{|c|}{$M_{\mathrm{C}} \sin i<42.5 M_{\mathrm{Jup}}$} & \multicolumn{3}{|c|}{$M_{\mathrm{C}} \sin i>42.5 M_{\mathrm{Jup}}$} & \multicolumn{2}{|c|}{$\mathrm{K}-\mathrm{S}$ test } \\
\hline & Range & Mean & Median & Range & Mean & Median & $D$ & $p$ \\
\hline$V(\mathrm{mag})$ & $3.29 / 10.82$ & 7.70 & 7.78 & $5.80 / 9.77$ & 7.70 & 7.68 & 0.24 & 0.40 \\
\hline Distance (pc) & $18.3 / 2174$ & 166.9 & 46.58 & $17.8 / 92.3$ & 44.4 & 44.9 & 0.26 & 0.31 \\
\hline Age (Gyr) & $0.66 / 11.48$ & 5.07 & 4.33 & $0.78 / 11.13$ & 5.31 & 5.29 & 0.25 & 0.43 \\
\hline $\operatorname{Mass}\left(M_{\odot}\right)$ & $0.40 / 2.53$ & 1.15 & 1.10 & $0.62 / 1.31$ & 0.97 & 0.99 & 0.31 & 0.16 \\
\hline$T_{\text {eff }}(\mathrm{K})$ & $4168 / 6163$ & 5330 & 5570 & $4860 / 6240$ & 5697 & 5795 & 0.34 & 0.08 \\
\hline SpType(\%) & \multicolumn{3}{|c|}{$13(\mathrm{~F}) ; 59(\mathrm{G}) ; 28(\mathrm{~K})$} & \multicolumn{3}{|c|}{$29(\mathrm{~F}) ; 57(\mathrm{G}) ; 14(\mathrm{~K})$} & & \\
\hline $\mathrm{LC}(\%)^{\dagger}$ & \multirow{2}{*}{\multicolumn{3}{|c|}{$\begin{array}{l}25(\mathrm{G}) ; 37.5(\mathrm{~S}) ; 37.5(\mathrm{MS}) \\
72(\mathrm{D}) ; 9(\mathrm{TD}) ; 19(\mathrm{R})\end{array}$}} & \multirow{2}{*}{\multicolumn{3}{|c|}{$\begin{array}{l}33 \text { (S); } 67 \text { (MS) } \\
75 \text { (D) } 10(\mathrm{TD}) \cdot 15(\mathrm{R})\end{array}$}} & & \\
\hline $\mathrm{D} / \mathrm{TD}(\%)^{\ddagger}$ & & & & & & 75 (D); 10 (TD); 15 (R) & & \\
\hline
\end{tabular}

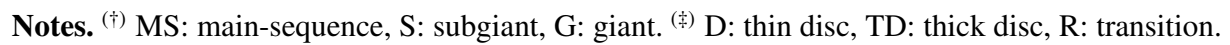

most likely to reflect the original metal content of the molecular cloud where the stars were born. The comparison is shown in Table 3. A series of two-sided Kolmogorov-Smirnov (K-S) tests (e.g. Peacock 1983) were performed to check whether the samples are likely or not drawn from the same parent population. The comparison shows that the samples both show similar distributions in brightness and age. The sample of SWBDs with $M_{\mathrm{C}} \sin i<42.5 M_{\text {Jup }}$ contains stars out to greater distances and slightly higher stellar masses than the SWBDs with
$M_{\mathrm{C}} \sin i>42.5 M_{\text {Jup }}$. Nevertheless, we note that the median distances for the two samples are quite similar and most of the stars, $\sim 75 \%$ with $M_{\mathrm{C}} \sin i<42.5 M_{\mathrm{Jup}}$, are within $92 \mathrm{pc}$ (the volume covered by the SWBDs with companions above $\left.42.5 M_{\text {Jup }}\right)$. As a further check, the metallicity-distance plane was explored and no metallicity difference was found between the SWBDs with $M_{\mathrm{C}} \sin i<42.5 M_{\mathrm{Jup}}$ located closer and farther than $92 \mathrm{pc}$. This potential bias is discussed in more detail in Sect. 3.1.6. Regarding the stellar mass, only four SWBDs with $M_{\mathrm{C}} \sin i<42.5 M_{\text {Jup }}$ 


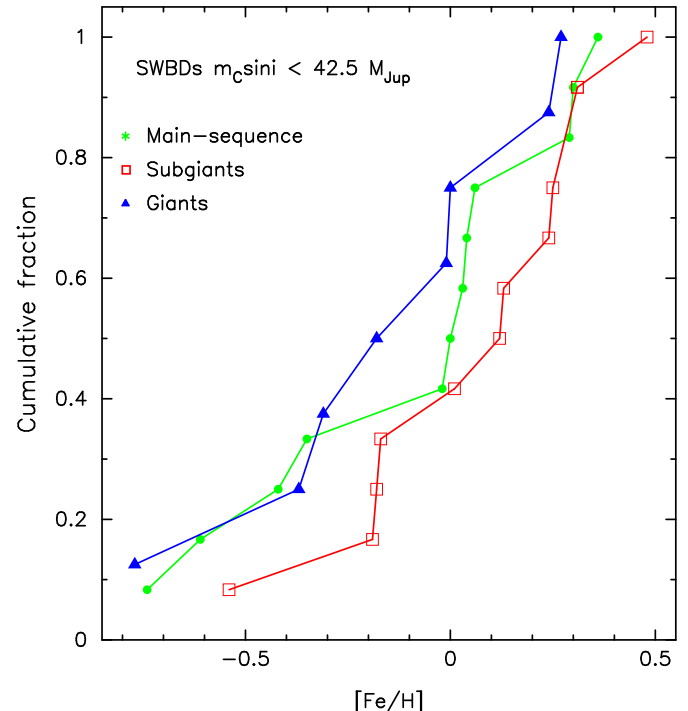

Fig. 3. Cumulative frequencies for SWBDs with companions in the mass range $M_{\mathrm{C}} \sin i<42.5 M_{\text {Jup }}$ according to their luminosity class.

show stellar masses higher than 1.4 $M_{\odot}$ (SWBDs with companions above $42.5 M_{\text {Jup }}$ cover up to $1.31 M_{\odot}$ ) showing a large range of metallicities (two stars have $[\mathrm{Fe} / \mathrm{H}] \sim+0.25$, one star shows solar metallicity, and the other is metal-poor with $[\mathrm{Fe} / \mathrm{H}] \sim$ $-0.30)$. We note that in the sample of SWBDs with $M_{\mathrm{C}} \sin i>$ 42.5 $M_{\text {Jup }}$ there are no giant stars; indeed, most of the stars $(\sim 67 \%)$ are on the main sequence. However, in its less massive counterpart sample, most of the stars are evolved, with about $62.5 \%$ of the stars in the giant and subgiant phase. This should be analysed carefully, since it has been shown that unlike their main-sequence counterparts, it is still unclear whether giant stars with planets show metal-enrichment (Sadakane et al. 2005; Schuler et al. 2005; Hekker \& Meléndez 2007; Pasquini et al. 2007; Takeda et al. 2008; Ghezzi et al. 2010; Maldonado et al. 2013; Mortier et al. 2013; Jofré et al. 2015; Reffert et al. 2015; Maldonado \& Villaver 2016). Furthermore, the abundance of some elements might be influenced by 3D or nLTE effects (e.g. Bergemann et al. 2011; Mashonkina et al. 2011). The metallicity distribution of SWBDs with $M_{\mathrm{C}} \sin i<42.5 M_{\text {Jup }}$ is shown in Fig. 3 where the stars are classified according to their luminosity class. The figure does not reveal any clear difference in metallicity between giants, subgiants, and main-sequence stars. We discuss this issue in more detail in Sect. 3.1.5.

\subsubsection{Kinematic biases}

Regarding kinematics, stars were classified as belonging to the thin/thick disc applying the methodology described in Bensby et al. (2003, 2005). For this purpose, we first computed the stellar spatial Galactic velocity components $(U, V, W)$ using the methodology described in Montes et al. (2001) and Maldonado et al. (2010), using the HIPPARCos parallaxes (van Leeuwen 2007), and Tycho-2 proper motions (Høg et al. 2000). Radial velocities were taken from the compilation of Kharchenko et al. (2007). The results show that most of the stars in both subsamples ( $\sim 72 \%$ and $\sim 75 \%$, respectively) should, according to their kinematics, belong to the thin $\operatorname{disc}^{9}$.

\footnotetext{
9 Our objective here is to discard the presence of a significant fraction of thick-disc stars within our samples (as these stars are expected to be relatively old, metal poor, and to show $\alpha$-enhancement) and not a
}

Table 4. $[\mathrm{Fe} / \mathrm{H}]$ statistics of the stellar samples.

\begin{tabular}{lcccccc}
\hline \hline Sample & Mean & Median & $\sigma$ & Min & Max & $N$ \\
\hline SWBDs & -0.10 & -0.03 & 0.32 & -0.92 & 0.48 & 53 \\
BDs $M_{\mathrm{C}} \sin i<42.5 M_{\text {Jup }}$ & -0.04 & 0.01 & 0.33 & -0.77 & 0.48 & 32 \\
BDs $M_{\mathrm{C}} \sin i>42.5 M_{\text {Jup }}$ & -0.18 & -0.11 & 0.28 & -0.92 & 0.17 & 21 \\
\hline SWOPs & -0.10 & -0.07 & 0.24 & -0.87 & 0.37 & 180 \\
SWGPs & 0.12 & 0.10 & 0.18 & -0.25 & 0.50 & 44 \\
SWLMPs & -0.03 & -0.01 & 0.23 & -0.38 & 0.42 & 17 \\
\hline
\end{tabular}

Another potential bias comes from the fact that several stars might harbour additional companions in the planetary range. Five SWBDs in the mass domain $M_{\mathrm{C}} \sin i<42.5 M_{\mathrm{Jup}}$ are known to host, in addition to a brown dwarf, at least one companion in the gas-giant planetary mass domain. These stars are HD 38529, HD 168443, HIP 5158, and HAT-P-13 (all with the planet closer to the star than the brown dwarf), and HD 202206, where the brown dwarf occupies the innermost orbit. We note that all these stars, except one (HD 168443), show significant positive metallicities. In order to test whether this fact could affect our results we compared the metallicity distribution of SWBDs with $M_{\mathrm{C}} \sin i<42.5 M_{\text {Jup }}$ when all the 32 stars with companions in this mass range are considered and when the four stars with possible additional planets are excluded. The results from the K-S test show that the two distributions are virtually equal with a probability of $\sim 99 \%$. Further analysis of this potential bias will be provided in Sect. 3.1.5. Finally, we note that only one star (BD+20 2457) harbours two companions in the brown dwarf regime.

\subsubsection{Metallicity distributions}

As mentioned before, 32 SWBDs are in the mass range $M_{\mathrm{C}} \sin i<42.5 M_{\text {Jup }}$ while 21 stars host BDs candidates with masses $M_{\mathrm{C}} \sin i>42.5 M_{\mathrm{Jup}}$. Some statistical diagnostics for the samples are summarised in Table 4, while their metallicity cumulative distribution functions are shown in Fig. 4. We also show the metallicity distribution of the whole sample of stars with brown dwarfs (i.e. all 53 stars with brown dwarf companions, SWBDs). In addition, several samples are overplotted for comparison: i) a sample of stars without known planetary companions (180 stars, SWOPs); ii) a sample of stars with known gasgiant planets (44 stars, SWGPs); and iii) a sample of stars with known low-mass planets ( $M_{\mathrm{p}} \sin i<30 M_{\oplus}, 17$ stars, SWLMPs). In order to be as homogeneous as possible, these comparison samples were taken from Maldonado et al. (2015) so their stellar parameters are determined with the same technique used in this work and using similar spectra.

There are a few interesting facts to be taken from the distributions shown in Fig. 4: i) SWBDs as a whole (magenta line) do not closely follow the trend of SWGPs (light blue line) that show metal enrichment; ii) considering the global metallicity distribution of SWBDs, there is a trend of SWBDs in the mass domain $M_{\mathrm{C}} \sin i<42.5 M_{\text {Jup }}$ (dark blue line) that have higher metallicities than SWBDs with $M_{\mathrm{C}} \sin i>42.5 M_{\text {Jup }}$ (red line); iii) for metallicities below approximately -0.20 , the metallicity distributions of SWBDs with masses above and below $42.5 M_{\text {Jup }}$ seem to follow a similar trend; iv) for higher metallicities, the distribution of SWBDs with companions in the mass range $M_{\mathrm{C}} \sin i<42.5 M_{\text {Jup }}$ clearly shifts towards higher metallicities

detailed thin/thick disc classification, which would require a detailed analysis of kinematics, ages, and abundances. 


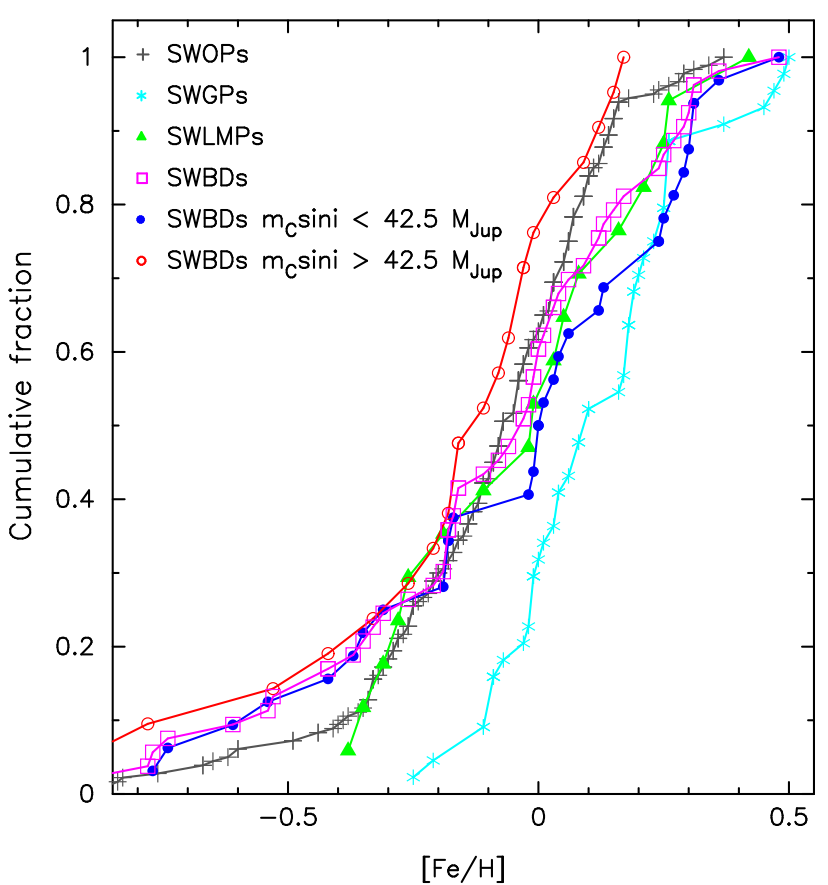

Fig. 4. Cumulative frequencies for the different samples studied in this work.

when compared with the distribution of SWBDs in the mass range $M_{\mathrm{C}} \sin i>42.5 M_{\mathrm{Jup}}$. We also note that at high metallicities (greater than +0.20 ), the metallicity distribution of SWBDs in the mass domain $M_{\mathrm{C}} \sin i<42.5 M_{\mathrm{Jup}}$ is similar to that of SWGPs.

These results can be compared with previous studies. Ma \& Ge (2014) and Mata Sánchez et al. (2014) found that the stars with brown dwarf companions do not show the metal-rich signature seen in stars hosting gas-giant planets. Furthermore, Ma \& Ge (2014) did not report metallicity differences between stars with BDs with minimum masses lower and higher than 42.5 $M_{\text {Jup }}$. We also note that Fig. 6 in Ma \& Ge (2014) shows results similar to ours. Around metallicities of $\sim+0.00$, stars with BDs with $M_{\mathrm{C}} \sin i<42.5 M_{\text {Jup }}$ tend to show higher metallicities reaching the metallicity distribution of stars with gas-giant planets at $[\mathrm{Fe} / \mathrm{H}] \sim+0.25$.

\subsubsection{Other chemical signatures}

In order to find differences in the abundances of other elements besides iron, Fig. 5 compares the cumulative distribution of $[\mathrm{X} / \mathrm{Fe}]$ between SWBDs with $M_{\mathrm{C}} \sin i$ below and above 42.5 $M_{\text {Jup }}$. Table 5 gives some statistic diagnostics, the results of a K-S test for each ion and also for $\left[\mathrm{X}_{\alpha} / \mathrm{Fe}\right],\left[\mathrm{X}_{\mathrm{Fe}} / \mathrm{Fe}\right]$, and $\left[\mathrm{X}_{\mathrm{vol}} / \mathrm{Fe}\right]$ (see definitions below). For Ca I, Sc I, Ti I, Cr I, and $\mathrm{Cr}$ II the distributions of the two samples seem to be quite similar. Indeed the probability that both samples come from the same distribution returned by the K-S tests for these ions is high $(>80 \%)$. On the other hand, for the abundances of Sc II, Mn I, $\mathrm{Ni}$ I, and $\mathrm{X}_{\mathrm{Fe}}$ the tests conclude that the two samples might be different.

In order to compare with the SWOP, SWGP, and SWLMP samples defined in Sect. 3.1.3, we grouped the ions into three categories: alpha elements, iron-peak elements, and volatile elements. For alpha and iron-peak elements we follow Mata Sánchez et al. (2014) and define $\left[\mathrm{X}_{\alpha} / \mathrm{Fe}\right]$ as the mean of the $[\mathrm{X} / \mathrm{Fe}]$ abundances of $\mathrm{Mg} \mathrm{I}, \mathrm{Si} \mathrm{I}, \mathrm{Ca} \mathrm{I}$, and Ti I, while $\left[\mathrm{X}_{\mathrm{Fe}} / \mathrm{Fe}\right]$ is defined as the mean of the Cr I, Mn I, Co I, and Ni I abundances.

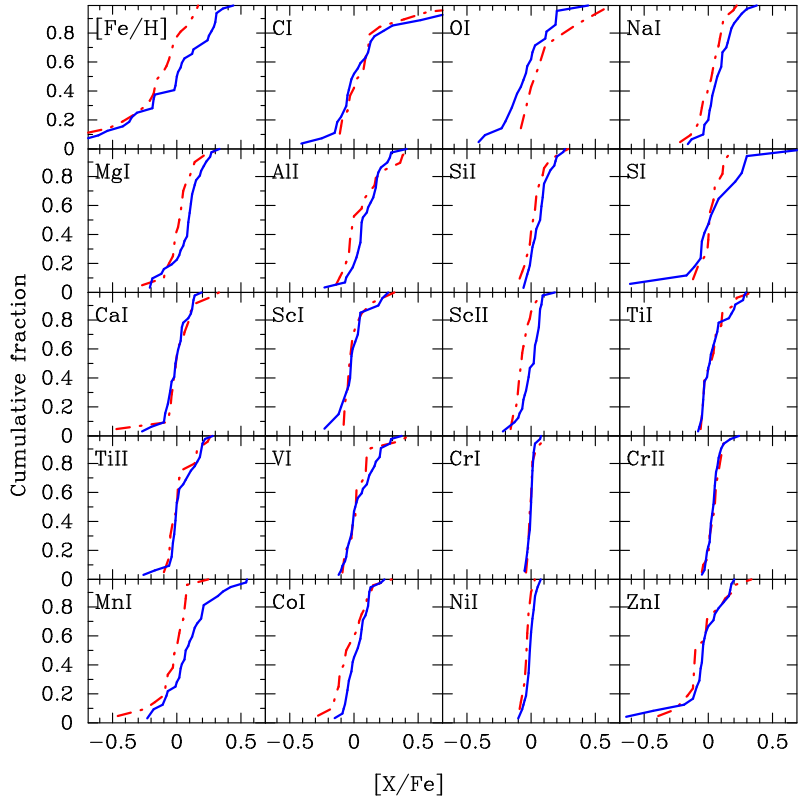

Fig. 5. $[\mathrm{X} / \mathrm{Fe}]$ cumulative fraction of SWBDs with $M_{\mathrm{C}} \sin i<42.5 M_{\text {Jup }}$ (blue continuous line) and $M_{\mathrm{C}} \sin i>42.5 M_{\text {Jup }}$ (red dash-dotted line).

Table 5. Comparison between the elemental abundances of stars with BDs with minimum masses $M_{\mathrm{C}} \sin i<42.5 M_{\mathrm{Jup}}$ and $M_{\mathrm{C}} \sin i>$ $42.5 M_{\text {Jup }}$.

\begin{tabular}{|c|c|c|c|c|c|c|c|}
\hline \multirow[t]{2}{*}[\mathrm{X}/\mathrm{Fe}]{} & \multicolumn{2}{|c|}{$M_{\mathrm{C}} \sin i<42.5 M_{\text {Jup }}$} & \multicolumn{2}{|c|}{$M_{\mathrm{C}} \sin i>42.5 M_{\mathrm{Jup}}$} & \multicolumn{3}{|c|}{$\mathrm{K}-\mathrm{S}$ test } \\
\hline & Median & $\sigma$ & Median & $\sigma$ & $D$ & $p$-value & $n_{\mathrm{eff}}$ \\
\hline C I & 0.00 & 0.29 & 0.07 & 0.32 & 0.19 & 0.80 & 11.15 \\
\hline O I & -0.04 & 0.21 & 0.06 & 0.26 & 0.38 & 0.34 & 5.25 \\
\hline $\mathrm{Na} \mathrm{I}$ & 0.10 & 0.12 & 0.03 & 0.11 & 0.26 & 0.32 & 12.35 \\
\hline Mg I & 0.10 & 0.14 & 0.03 & 0.13 & 0.40 & 0.03 & 12.16 \\
\hline $\mathrm{Al} \mathrm{I}$ & 0.07 & 0.13 & 0.00 & 0.17 & 0.35 & 0.09 & 11.48 \\
\hline Si I & 0.08 & 0.08 & 0.03 & 0.10 & 0.29 & 0.19 & 12.68 \\
\hline S I & 0.02 & 0.30 & 0.01 & 0.09 & 0.29 & 0.53 & 6.68 \\
\hline $\mathrm{Ca} \mathrm{I}$ & 0.01 & 0.10 & 0.01 & 0.15 & 0.13 & 0.97 & 12.68 \\
\hline Sc I & -0.02 & 0.12 & -0.01 & 0.12 & 0.20 & 0.87 & 7.88 \\
\hline Sc II & 0.03 & 0.09 & -0.07 & 0.07 & 0.45 & 0.01 & 12.68 \\
\hline Ti I & 0.02 & 0.11 & 0.04 & 0.11 & 0.12 & 0.99 & 12.68 \\
\hline Ti II & 0.00 & 0.12 & 0.01 & 0.10 & 0.21 & 0.62 & 12.31 \\
\hline $\mathrm{V}_{\mathrm{I}}$ & 0.02 & 0.13 & 0.02 & 0.13 & 0.20 & 0.63 & 12.68 \\
\hline Cr I & 0.00 & 0.03 & 0.00 & 0.04 & 0.17 & 0.85 & 12.68 \\
\hline Cr II & 0.04 & 0.06 & 0.06 & 0.05 & 0.15 & 0.94 & 12.16 \\
\hline Mn I & 0.09 & 0.19 & 0.00 & 0.15 & 0.45 & 0.01 & 12.68 \\
\hline Co I & 0.03 & 0.09 & -0.03 & 0.14 & 0.37 & 0.05 & 12.16 \\
\hline Ni I & 0.00 & 0.04 & -0.03 & 0.03 & 0.48 & $<0.01$ & 12.68 \\
\hline Zn I & -0.04 & 0.19 & -0.10 & 0.17 & 0.36 & 0.09 & 11.20 \\
\hline $\mathrm{X}_{\alpha}$ & 0.04 & 0.09 & 0.01 & 0.10 & 0.23 & 0.45 & 12.68 \\
\hline $\mathrm{X}_{\mathrm{Fe}}$ & 0.03 & 0.06 & -0.03 & 0.04 & 0.47 & $<0.01$ & 12.68 \\
\hline $\mathrm{X}_{\mathrm{vol}}$ & 0.05 & 0.15 & 0.03 & 0.12 & 0.19 & 0.73 & 12.68 \\
\hline
\end{tabular}

We define the mean volatile abundance, $\left[\mathrm{X}_{\mathrm{vol}} / \mathrm{Fe}\right]$, as the mean of the $[\mathrm{X} / \mathrm{Fe}]$ values of the elements with a condensation temperature, $T_{\mathrm{C}}$, lower than $900 \mathrm{~K}$, namely C I, O I, S I, and Zn I. Although $\mathrm{Na} I$ has a $T_{\mathrm{C}}$ slightly above $900 \mathrm{~K}$ we include it in the group of volatiles to account for the fact that for some stars the abundances of some volatiles were not obtained. It is important to mention at this point that the abundances in the comparison samples for this work were derived in a similar way by Maldonado et al. (2015) and Maldonado \& Villaver (2016).

The different cumulative functions are shown in Fig. 6. Interestingly, the figure reveals a tendency of SWBDs in the low-mass 

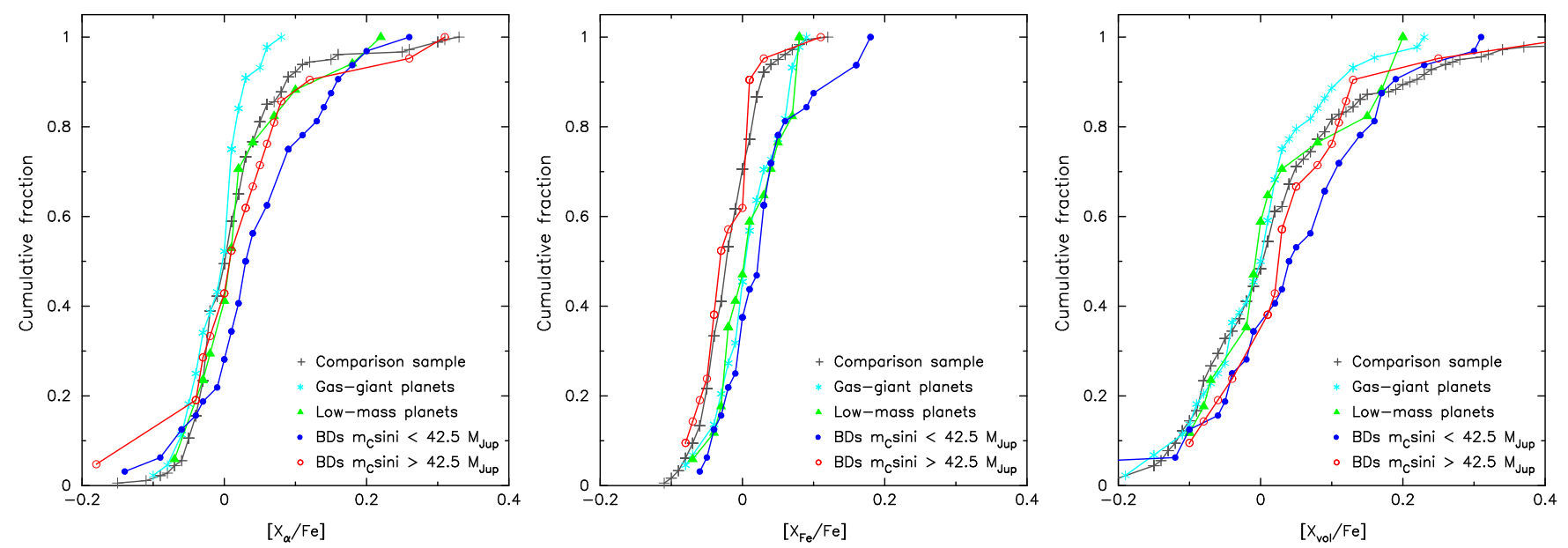

Fig. 6. Cumulative frequencies of $\left[\mathrm{X}_{\alpha} / \mathrm{Fe}\right](l e f t),\left[\mathrm{X}_{\mathrm{Fe}} / \mathrm{Fe}\right]\left(\right.$ middle), and $\left[\mathrm{X}_{\mathrm{vol}} / \mathrm{Fe}\right]($ right $)$ for the different samples studied in this work.

Table 6. Comparison between the abundances of stars with BDs with minimum masses $M_{\mathrm{C}} \sin i<42.5 M_{\text {Jup }}$ and the SWOP, SWLMP, and SWGP samples.

\begin{tabular}{lcccccc}
\hline \hline & \multicolumn{2}{c}{$\begin{array}{c}\text { Stars without } \\
\text { planets }\end{array}$} & \multicolumn{2}{c}{$\begin{array}{c}\text { Stars with low- } \\
\text { mass planets }\end{array}$} & \multicolumn{2}{c}{$\begin{array}{c}\text { Stars with gas- } \\
\text { giant planets }\end{array}$} \\
{$[\mathrm{X} / \mathrm{Fe}]$} & $D$ & $p$-value & $D$ & $p$-value & $D$ & $p$-value \\
\hline $\mathrm{X}_{\alpha}$ & 0.25 & 0.05 & 0.30 & 0.23 & 0.43 & $<0.01$ \\
$\mathrm{X}_{\mathrm{Fe}}$ & 0.40 & $<0.01$ & 0.19 & 0.78 & 0.17 & 0.63 \\
$\mathrm{X}_{\mathrm{vol}}$ & 0.21 & 0.16 & 0.30 & 0.21 & 0.31 & 0.04 \\
\hline
\end{tabular}

Notes. $n_{\mathrm{eff}} \sim 27.2$ (SWOPs); 11.1 (SWLMPs); 18.5 (SWGPs).

domain to have slightly higher abundances than the rest of the samples in all categories. In order to test this tendency, the SWBDs with companions in the mass range $M_{\mathrm{C}} \sin i<42.5 M_{\mathrm{Jup}}$ were compared (by means of a K-S test) with the rest of the samples. The results are provided in Table 6 .

Regarding $\alpha$ elements, the sample of stars with low-mass BDs companions does not seem to be different from the SWOP and SWLMP samples, although we note the low $p$-value of 0.05 when compared with the SWOP sample. The K-S test suggests, however, that the sample differs from the sample of stars harbouring gas-giant planets ( $p$-value $<0.01)$. Since this is a surprising result, we have checked if our SWGP sample occupies the same place in the $\left[\mathrm{X}_{\alpha} / \mathrm{Fe}\right]$ vs. $[\mathrm{Fe} / \mathrm{H}]$ plot as other samples in the literature. For this check we have taken the data from Adibekyan et al. (2012) and find consistent results, i.e. our SWGP and the stars with giant planets from Adibekyan et al. (2012) both tend to show high metallicity values and rather low $\left[\mathrm{X}_{\alpha} / \mathrm{Fe}\right]$ values. The most significant differences appear when considering the iron-peak elements. In this case the sample of SWBDs in the low-mass companion range seems to be shifted towards higher metallicities when compared with the SWOPs. No statistically significant differences are found when considering the volatile elements, although we note that in the comparison with the SWGPs the $p$-value is relatively low (only 0.04).

We therefore conclude that the SWBD sample with companions in the mass range $M_{\mathrm{C}} \sin i<42.5 M_{\mathrm{Jup}}$ may differ from the SWOPs in iron-peak elements, but also from the GWPs when considering $\alpha$ elements.

\subsubsection{Presence of red giants and additional planetary companions}

As already pointed out, $25 \%$ of the stars in the sample with low-mass brown dwarf companions are red giants. To check for possible biases we repeated the comparison of the abundance properties $\left([\mathrm{Fe} / \mathrm{H}],\left[\mathrm{X}_{\alpha} / \mathrm{Fe}\right],\left[\mathrm{X}_{\mathrm{Fe}} / \mathrm{Fe}\right],\left[\mathrm{X}_{\mathrm{vol}} / \mathrm{Fe}\right]\right)$ of the SWBD with companions with masses above and below $42.5 M_{\text {Jup }}$, excluding from the analysis all stars classified as giants. The results are shown in Table 7 where the new analysis is compared with the previous one. It can be seen that the results do not change in a significant way. For example, for $[\mathrm{Fe} / \mathrm{H}]$ the $p$-value changes from 0.08 to 0.05 , while when considering $\left[\mathrm{X}_{\mathrm{Fe}} / \mathrm{Fe}\right]$ it moves from less than 0.01 to 0.04 . Although the threshold of 0.02 on the $p$-value is usually assumed to consider statistical significance when interpreting the results from the K-S tests, we note that a $p$-value of 0.04 is still very low. We conclude that the presence of giant stars in the SWBDs with companions below $42.5 M_{\text {Jup }}$ does not introduce any significant bias in the comparisons performed in this work.

However, the results might change if in addition to the giant stars we also exclude the subgiant stars (from both SWBDs subsamples). In this case, the $p$-value for $[\mathrm{Fe} / \mathrm{H}]$ increases from 0.08 to 0.24 , while for $\left[\mathrm{X}_{\mathrm{Fe}} / \mathrm{Fe}\right]$ it goes from less than 0.01 to a value of 0.64 . This is in contrast to what we found when excluding only the giant stars from the analysis and may, at least partially, be due to the significant reduction of the sample size. We note that by excluding both giant and subgiant stars from the analysis we are reducing the sample to approximately half the original size.

Finally, we analyse the results when the stars with additional companions in the planetary mass are excluded (all of them in the sample of stars with low-mass brown dwarfs; see Table 7). In this case the significance of a possible metallicity difference between SWBDs with companions above and below $42.5 M_{\text {Jup }}$ diminishes (the $p$-value changes from 0.08 to 0.31 ). The $p$-value for $\left[\mathrm{X}_{\mathrm{Fe}} / \mathrm{Fe}\right]$ also rises a bit from less than 0.01 to 0.03 . We again conclude that no significant bias is introduced by the five SWBDs with companions below $42.5 M_{\text {Jup }}$ which, in addition to brown dwarf companions, also harbours companions in the gas-giant planetary mass domain.

\subsubsection{Stellar distance bias}

As shown in Sect. 3.1.1 our sample contains several stars far from the solar neighbourhood including objects up to distances of $2174 \mathrm{pc}$. However, most of the studies of the solar neighbourhood are volume limited. In particular it should be noticed that as the distance increases astrometry becomes difficult, and therefore only minimum masses are available. 
Table 7. Comparison between the abundances of stars with BDs with minimum masses $M_{\mathrm{C}} \sin i>42.5 M_{\mathrm{Jup}}$ and stars with BD companions with minimum masses in the range $M_{\mathrm{C}} \sin i<42.5 M_{\text {Jup }}$ performed in Sect. 3.1.5, see text for details.

\begin{tabular}{lcccccccc}
\hline \hline & \multicolumn{2}{c}{$\begin{array}{c}\text { All } \\
\text { stars }\end{array}$} & \multicolumn{2}{c}{$\begin{array}{c}\text { Without } \\
\text { giant stars }\end{array}$} & \multicolumn{2}{c}{$\begin{array}{c}\text { Without } \\
\text { subgiant stars }\end{array}$} & \multicolumn{2}{c}{$\begin{array}{c}\text { Without stars with } \\
\text { gas-giant planets }\end{array}$} \\
\hline & $D$ & $p$-value & $D$ & $p$-value & $D$ & $p$-value & $D$ & $p$-value \\
\hline$[\mathrm{Fe} / \mathrm{H}]$ & 0.34 & 0.08 & 0.39 & 0.05 & 0.38 & 0.24 & 0.27 & 0.31 \\
$\mathrm{X}_{\alpha}$ & 0.23 & 0.45 & 0.15 & 0.95 & 0.26 & 0.70 & 0.26 & 0.33 \\
$\mathrm{X}_{\mathrm{Fe}}$ & 0.47 & $<0.01$ & 0.40 & 0.04 & 0.27 & 0.64 & 0.40 & 0.03 \\
$\mathrm{X}_{\mathrm{vol}}$ & 0.19 & 0.73 & 0.18 & 0.83 & 0.35 & 0.35 & 0.24 & 0.46 \\
\hline
\end{tabular}

Notes. $n_{\mathrm{eff}} \sim 12.7$ (all stars); $\sim 11.2$ (without giant stars); $\sim 6.5$ (without subgiant/giant stars); $\sim 11.8$ (without planet hosts).

Table 8. Comparison between the abundances of stars with BDs with minimum masses $M_{\mathrm{C}} \sin i>42.5 M_{\mathrm{Jup}}$ and stars with BD companions with minimum masses in the range $M_{\mathrm{C}} \sin i<42.5 M_{\text {Jup }}$ performed in Sect. 3.1.6, see text for details.

\begin{tabular}{lcccc}
\hline \hline & \multicolumn{2}{c}{$d<50 \mathrm{pc}$} & \multicolumn{2}{c}{$d<75 \mathrm{pc}$} \\
\hline & $D$ & $p$-value & $D$ & $p$-value \\
\hline$[\mathrm{Fe} / \mathrm{H}]$ & 0.48 & 0.04 & 0.43 & 0.03 \\
$\mathrm{X}_{\alpha}$ & 0.27 & 0.55 & 0.17 & 0.90 \\
$\mathrm{X}_{\mathrm{Fe}}$ & 0.38 & 0.16 & 0.39 & 0.06 \\
$\mathrm{X}_{\mathrm{vol}}$ & 0.32 & 0.34 & 0.15 & 0.97 \\
\hline
\end{tabular}

Notes. $n_{\text {eff }} \sim 7.7(d<50 \mathrm{pc}) ; \sim 10.2(d<75 \mathrm{pc})$.

In order to check whether our results are affected by having stars at relatively large distances, we repeated the statistical analysis performed earlier, but only considered the stars with distances lower than $50 \mathrm{pc}$ and the stars located within $75 \mathrm{pc}$. Approximately $60 \%$ of our stars are within $50 \mathrm{pc}$, while this percentage increases to $\sim 77 \%$ for a distance of $75 \mathrm{pc}$. The results are shown in Table 8, and can be compared with the first column of Table 7. We find that the values of the K-S statistic $(D)$ do not change in a significant way. Regarding the $p$-values, only those corresponding to $\mathrm{X}_{\alpha}$ and $\mathrm{X}_{\mathrm{vol}}$ seem to increase when the SWBD sample is limited to stars within $75 \mathrm{pc}$. The interpretation, however, does not change: differences in metallicity and iron-peak elements seem to be present (note the very low $p$-values) between SWBDs with companions with minimum masses above and below $42.5 M_{\text {Jup }}$ irrespectively of whether all stars or a volumelimited sample is considered.

\subsubsection{Minimum and true masses}

Another source of bias that might be influencing this study is that for most of our SWBDs only the minimum mass of the mass brown candidate is known. This is an important effect as the distribution of minimum masses given by radial velocity surveys of brown dwarfs might be less indicative of a true substellar mass than for objects in the planetary mass regime (see e.g. Stevens \& Gaudi 2013)

Given that we do not have information regarding the inclination angle of the BD stellar systems we have tried to account for this effect by considering a series of scenarios: i) a "pessimistic" scenario in which all our stars are seen at very low inclinations (15 degrees); ii) a "favourable" case in which all our stars are seen at high inclinations (85 degrees); iii) a random distribution $P(i)$ for the orientation of the inclination expressed as $P(i) d i=\sin (i) d i$; and iv) the case where a series of $10^{4}$ simulations are performed with random inclinations for each star. In all cases we keep the "true" brown-dwarf masses when available. We note that in case iii) the average value of $\sin i$ assuming a random inclination, $\langle\sin (i)\rangle=0.785$, is used to estimate the mass of the brown dwarfs. Although more complex algorithms exist to compute the probability distribution for $\sin (i)$, it has been shown that the use of the average value produces similar results for small number statistics (see Grether \& Lineweaver 2006, and references therein).

Table 9 shows the results from the K-S test for all these scenarios. The conclusion is that unless we are in the unlikely case that most of the stars are seen at very low inclinations angles (case i) the results do not change in a significant way (compare with first column in Table 7). In particular, we note that the results from the scenario iii) are very similar to the results where no inclination is assumed. The results from the simulations performed in case iv) are somewhat inconclusive given the wide spread found for the $p$-values.

\subsection{Abundances and brown dwarf properties}

A study of the possible relationships between stellar metallicity and the properties of the BD companions was also performed. Figure 7 shows the stellar metallicity as a function of the BD minimum mass, period, and eccentricity. The figure does not reveal any clear correlation between the metallicity and the BDs properties.

The figure clearly shows the brown dwarf desert, as nearly $81.5 \%$ of the BDs have periods longer than 200 days. This is in sharp contrast with the presence of a significant number of gasgiant and low-mass planets at short periods. Among the stars with periods shorter than 200 days, we note that only three BDs are in the mass range $M_{\mathrm{C}} \sin i<42.5 M_{\text {Jup }}$. Regarding the eccentricities, we note that BDs in the mass range $M_{\mathrm{C}} \sin i<42.5 M_{\text {Jup }}$ tend to show low values; $\sim 70 \%$ of the BDs in this mass range have eccentricities lower than 0.5 .

Figure 8 shows the cumulative distribution function of periods (left) and eccentricities (right) for SWBDs according to the mass of the companion. The analysis of the periods reveals that for periods shorter than $\sim 1000$ days, the sample of SWBDs with $M_{\mathrm{C}} \sin i>42.5 M_{\mathrm{Jup}}$ shows shorter values than SWBDs with less massive companions. A K-S test gives a probability that both samples show the same period distribution of $\sim 6 \%$. The sample of SWBDs with companions more massive than $42.5 M_{\text {Jup }}$ clearly shows higher eccentricities than the SWBDs with companions below $42.5 M_{\text {Jup }}$, at least up to a value of $\sim 0.6$. The K-S test on the eccentricity values suggests that the two samples are statistically different ( $p$-value $\sim 10^{-16}$ ). These results are consistent with the findings of Ma \& Ge (2014). 
Table 9. Comparison between the abundances of stars with BDs with masses above and below $42.5 M_{\text {Jup }}$ performed in Sect. 3.1.7, see text for details.

\begin{tabular}{lcccccccc}
\hline \hline & \multicolumn{2}{c}{$i=15^{\circ}$} & \multicolumn{2}{c}{$i=85^{\circ}$} & \multicolumn{2}{c}{$\langle\sin (i)\rangle$} & \multicolumn{2}{c}{$i$ random $^{\dagger}$} \\
\hline & $D$ & $p$-value & $D$ & $p$-value & $D$ & $p$-value & $D$ & $p$-value \\
\hline$[\mathrm{Fe} / \mathrm{H}]$ & 0.31 & 0.52 & 0.34 & 0.08 & 0.34 & 0.11 & $0.32 \pm 0.09$ & $0.32 \pm 0.27$ \\
$\mathrm{X}_{\alpha}$ & 0.29 & 0.61 & 0.23 & 0.45 & 0.18 & 0.80 & $0.23 \pm 0.07$ & $0.62 \pm 0.29$ \\
$\mathrm{X}_{\mathrm{Fe}}$ & 0.37 & 0.31 & 0.47 & $<0.01$ & 0.44 & $<0.01$ & $0.37 \pm 0.10$ & $0.22 \pm 0.24$ \\
$\mathrm{X}_{\text {vol }}$ & 0.36 & 0.35 & 0.19 & 0.73 & 0.20 & 0.69 & $0.24 \pm 0.07$ & $0.58 \pm 0.27$ \\
\hline
\end{tabular}

Notes. $n_{\text {eff }} \sim 5.9\left(i=15^{\circ}\right) ; \sim 12.7\left(i=85^{\circ}\right) ; \sim 11.8(\langle\sin (i)\rangle) ; \sim 9.5 \pm 0.9\left(i\right.$ random). ${ }^{(\dagger)}$ The mean values of $10^{4}$ simulations are shown with their corresponding standard deviations.
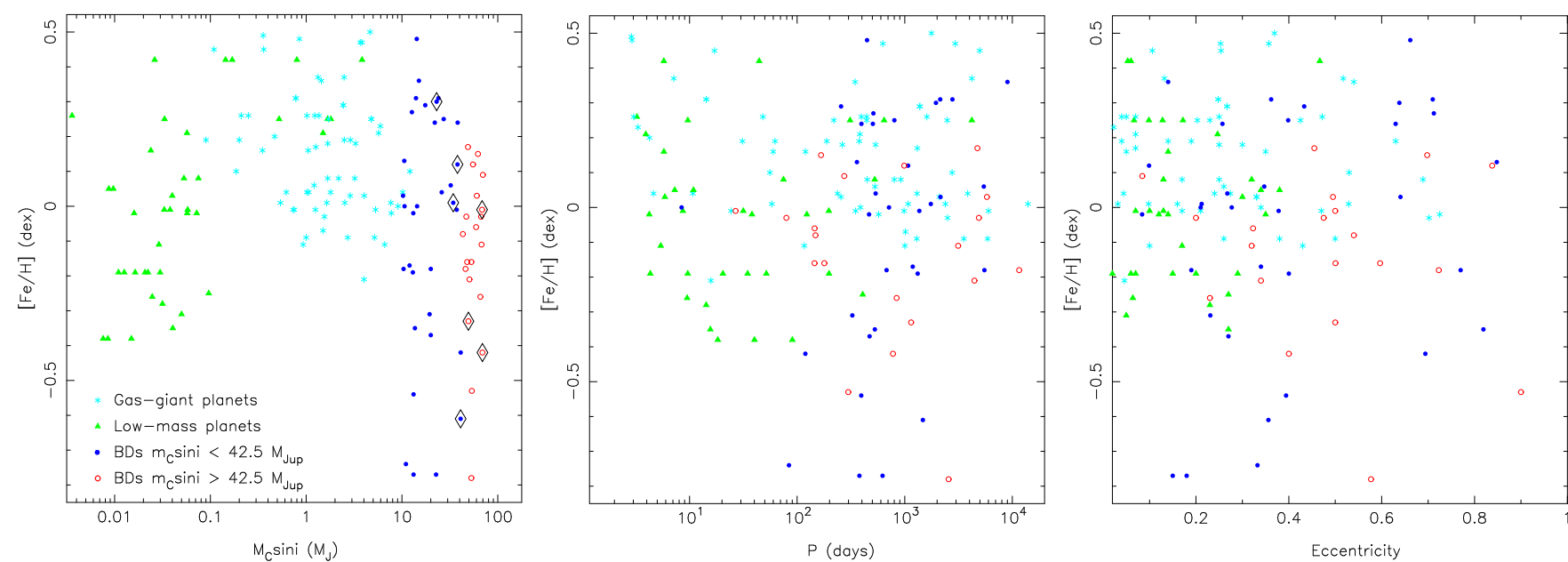

Fig. 7. Stellar metallicity as a function of the brown dwarf or planetary companion properties. The colours and symbols are the same as in the previous figures. Diamonds indicate brown dwarfs with "true" mass determinations. Left: minimum mass; middle: period; right: eccentricity.
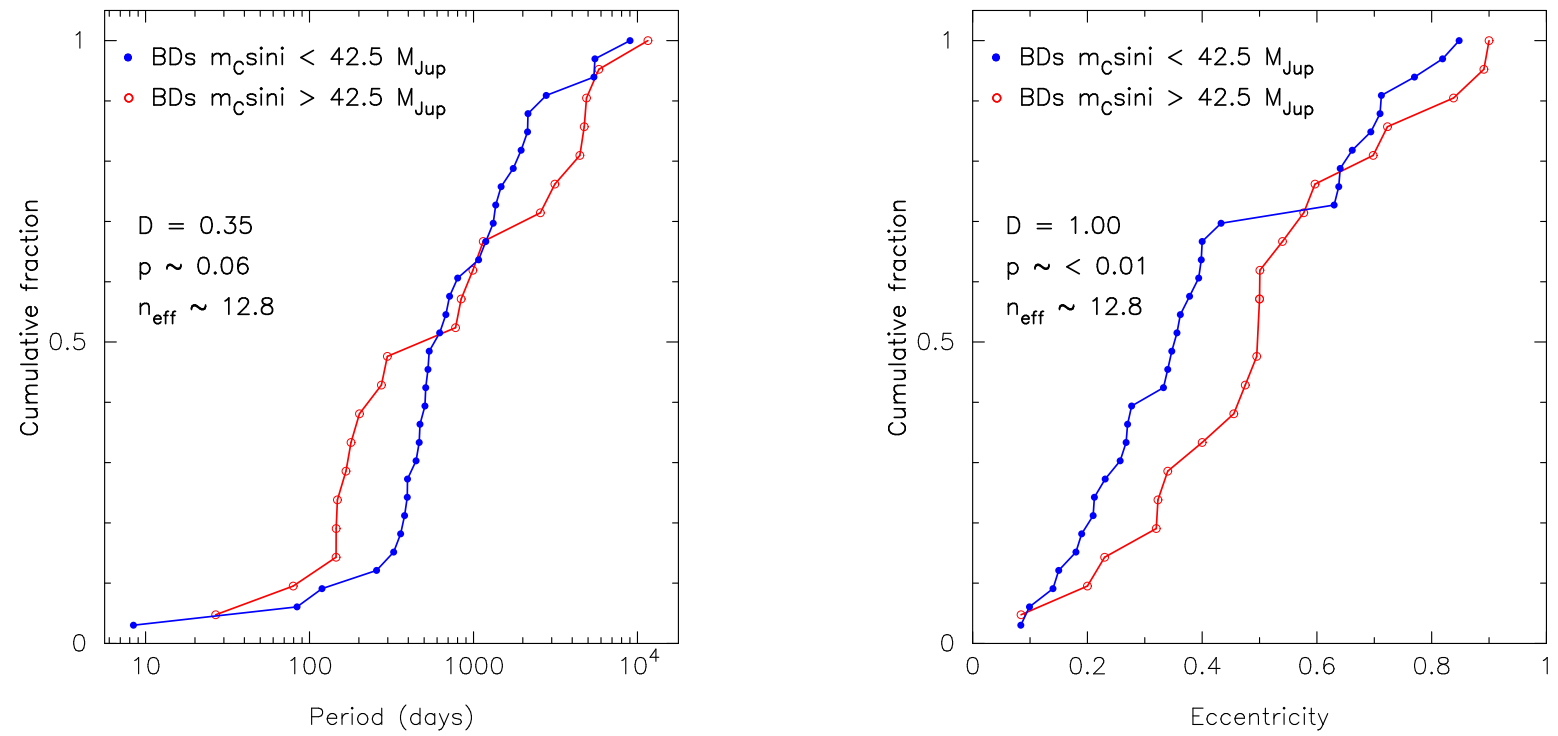

Fig. 8. Cumulative distribution function of periods (left) and eccentricities (right) for SWBDs with $M_{\mathrm{C}} \sin i<42.5 M_{\mathrm{Jup}}$ and $M_{\mathrm{C}} \sin i>42.5 M_{\mathrm{Jup}}$.

\section{Discussion}

The existence of the brown dwarf desert has lead to numerous theories about whether brown dwarfs form like low-mass stars, like giant-planets, or by entirely different mechanisms (see e.g. Chabrier et al. 2014, for a recent review). The first observational results of this work suggest that BDs form in a different way from gas-giant planets (if metallicity, as is often assumed, traces the formation mechanism), as it is clear from Fig. 4 that SWBDs do not follow the well-known gas-giant planet metallicity correlation. This can also be seen in the left panel of Fig. 7.
In a recent work, Ma \& Ge (2014) show that massive and low-mass brown dwarfs have significantly different eccentricity distributions. This difference is also seen in our sample. In particular, the authors note that BDs with masses above $42.5 M_{\text {Jup }}$ have an eccentricity distribution consistent with that of binaries. This result alone could be interesting in that it reveals clues regarding the formation mechanism of brown dwarfs. However, based on the eccentricity distribution alone we cannot directly infer that BDs and low-mass stars are formed via the same process, i.e. fragmentation of a molecular cloud. Different formation mechanisms can lead to similar eccentricity distributions when 
subject to particular dynamical histories. What adds support to the hypothesis of a similar formation process is that our analysis does not reveal any hint of metal enrichment in the stars with brown dwarf companions with masses above $42.5 \mathrm{M}_{\mathrm{Jup}}$. Moreover, in all the analyses performed in this work, SWBDs with masses above $42.5 M_{\text {Jup }}$ follow similar distributions to those of SWOPs or SWLMPs (see Figs. 4 and 6), suggesting a nonmetallicity/abundance dependent formation.

It has been shown that BDs can be formed via gravitational instability in the outer parts (>100 au) of massive circumstellar discs (with stellar/disc mass ratios of the order of unity) (Stamatellos \& Whitworth 2009). The eccentricities were found to be very high as a result of this formation process, but the authors noted that it might be an artefact of the simulations that do not include tidal interactions with the gas disc. BDs in the so-called ejection scenario are formed by gravoturbulent fragmentation of collapsing pre-stellar cores; due to dynamical interactions, these BDs end-up being ejected from the cloud, terminating the accretion process (see e.g. Bate 2009a,b). In this scenario eccentricities are not expected to populate the high end of the eccentricity distribution.

Support for a different formation mechanism for low-mass and massive brown dwarfs came from the chemical analysis performed in this work. Our results show a tendency of SWBDs with masses below $42.5 M_{\text {Jup }}$ to have slightly higher metallicities and abundances (especially $\mathrm{X}_{\mathrm{Fe}}$ ) than SWBDs with masses above 42.5 $M_{\text {Jup }}$ (see Figs. 4-6), although with low statistical significance (Table 5). We should note, however, that the results for $\mathrm{X}_{\mathrm{Fe}}$ are statistically significant. These results can be compared with the recent work by Mata Sánchez et al. (2014), where the authors already note the possible higher $\alpha$-element and Fepeak abundances in the stars hosting brown dwarfs with masses below 42.5 $\mathrm{M}_{\text {Jup }}$ (in comparison with those hosting more massive brown dwarfs); however, these authors do not directly test the significance of these possible trends. Furthermore, their sample of SWBDs is significantly smaller than the one analysed in this work.

If low-mass brown dwarfs were formed by core-accretion rather by the gravitational instability mechanism, stars hosting low-mass brown dwarfs should show the metal-rich signature seen in gas-giant planetary hosts (e.g. Gonzalez 1997; Santos et al. 2004; Fischer \& Valenti 2005). It is clear from Fig. 4, that SWBDs with masses below $42.5 M_{\text {Jup }}$ show lower metallicities than SWGPs. A K-S test confirms that the two samples are different $\left(D \sim 0.33, p\right.$-value $\left.\sim 0.03, n_{\text {eff }} \sim 18.5\right)$. Only for metallicities above $\sim+0.20$ dex does the metallicity distribution of SWBDs with masses below 42.5 $M_{\text {Jup }}$ approach the distribution of the SWGP sample. As already discussed, this might be affected by the presence of additional planetary companions in some SWBDs. Indeed, when the stars with additional planets are removed from the SWBD sample, the higher metallicities of SWGPs becomes more significant $(D \sim 0.40, p$-value $\sim 0.007$, $n_{\text {eff }} \sim 16.7$ ).

Low-mass brown dwarfs might form in self-gravitating protostellar discs (Rice et al. 2003b), a fast mechanism that does not require the previous formation of a rocky core and is therefore independent of the stellar metallicity (Boss 2002, 2006). The simulations by Rice et al. (2003b) shows that the fragmentation of an unstable protostellar disc produces a large number of substellar objects, although most of them are ejected from the system. The remaining objects are typically either a very massive planet or a low-mass brown dwarf, having long periods and high eccentricities ${ }^{10}$. It is possible that four of the systems discussed in Sect. 3.1.1 (namely HD 38529, HD 168443, HIP 5158, and HAT-P-13) with a planet in an inner orbit and a brown dwarf at a larger distance formed in this way, as did the two brown dwarf system around the metal-poor star BD+20 $2457([\mathrm{Fe} / \mathrm{H}]=$ -0.77 dex). The case of the system around HD 202206 (also mentioned in Sect. 3.1.1) might need further discussion as the brown dwarf has an inner orbit to the planet one.

Rice et al. (2003a) also shows that as the disc mass increases various effects might act to make the disc more unstable. A relationship between the disc mass and the stellar mass of the form $M_{\text {disc }} \propto M_{\star}^{1.2}$ have been suggested (Alibert et al. 2011) to explain the observed correlation between mass-accretion rate scales and stellar mass in young low-mass objects (Muzerolle et al. 2003; Natta et al. 2004; Mendigutía et al. 2011, 2012). The fact that more massive stars might have more massive and more unstable discs might explain the presence of a relatively large number of low-mass BDs around evolved stars (subgiants and red giants) as it has been shown that these stars are indeed more massive (e.g. Maldonado et al. 2013).

So our results on the chemical analysis of BDs suggest that at low metallicities the dominant mechanism of BD formation is compatible with gravitational instability in massive discs or gravoturbulent fragmentation of collapsing pre-stellar cores (i.e. physical mechanisms that do not depend on the metal content of the cloud). The fact that we observed differences in the metal content for low- and high-mass BDs at high metallicities could indicate different mechanisms operating at different efficiencies. Core accretion might favour the formation of low-mass BDs at high metallicities even at low disc masses, while it might inhibit the formation of massive BDs as not enough mass reservoir is available in the disc. For low-mass BDs orbiting high-metallicity host stars the core accretion model might become efficient and favour the formation of BDs even at lower disc masses and inhibit the formation of BDs with higher masses (not enough mass in the disc). It is important to note that different BD/planet formation mechanisms can operate together and do not have to be exclusive of each other.

\section{Conclusions}

In this work, a detailed chemical analysis of a large sample of stars with brown dwarfs has been presented. The sample has been analysed taking into account the presence of massive $\left(M_{\mathrm{C}} \sin i>42.5 M_{\mathrm{Jup}}\right)$ and low-mass brown dwarfs $\left(M_{\mathrm{C}} \sin i<\right.$ $42.5 M_{\text {Jup }}$ ) companions. Before comparing the two subsamples, a detailed analysis of their stellar properties was performed to control any possible bias affecting our results. The chemical abundances of the SWBDs have also been compared to those of stars with known planetary companions, and with a sample of stars without planets.

Our results show that SWBDs do not follow the well-known gas-giant metallicity correlation seen in main-sequence stars with planets. A tendency of SWBDs with substellar companions in the mass range $M_{\mathrm{C}} \sin i>42.5 M_{\mathrm{Jup}}$ to have slightly higher metallicities and abundances than those of SWBDs with substellar companions in the mass range $M_{\mathrm{C}} \sin i<42.5 M_{\mathrm{Jup}}$ seems to be present in the data. However, its statistical significance is rather low. We also confirm possible differences between SWBDs with substellar companions with masses above

${ }^{10}$ This is not at odds with our results from Fig. 8, right panel, as usually eccentricities of the order of 0.2 are considered as "high" in the literature. 
and below 42.5 $M_{\text {Jup }}$ in terms of periods and eccentricities. All this observational evidence suggests that the efficiencies of the different formation mechanisms may differ for low-mass and high-mass brown dwarfs.

Our results are well described in a scenario in which highmass brown dwarfs mainly form in the same way as low-mass stars (by the fragmentation of a molecular cloud). Our analysis shows that at high metallicities the core-accretion model might be the mechanism for the formation of low-mass BDs. On the other hand, it seems reasonable that the most suitable scenario for the formation of low-metallicity, low-mass BDs is gravitational instability in turbulent protostellar discs since this mechanism is known to be independent of the stellar metallicity.

Acknowledgements. This research was supported by the Italian Ministry of Education, University, and Research through the PREMIALE WOW 2013 research project under grant Ricerca di pianeti intorno a stelle di piccola massa. E.V. acknowledges support from the On the rocks project funded by the Spanish Ministerio de Economía y Competitividad under grant AYA2014-55840-P. Carlos Eiroa is acknowledged for valuable discussions. We sincerely appreciate the careful reading of the manuscript and the constructive comments of an anonymous referee.

\section{References}

Adibekyan, V. Z., Sousa, S. G., Santos, N. C., et al. 2012, A\&A, 545, A32 Alibert, Y., Mordasini, C., \& Benz, W. 2004, A\&A, 417, L25 Alibert, Y., Mordasini, C., \& Benz, W. 2011, A\&A, 526, A63

Allende Prieto, C., Lambert, D. L., \& Asplund, M. 2001, ApJ, 556, L63

Arenou, F., Grenon, M., \& Gomez, A. 1992, A\&A, 258, 104

Baranne, A., Queloz, D., Mayor, M., et al. 1996, A\&AS, 119, 373

Bate, M. R. 2009a, MNRAS, 392, 590

Bate, M. R. 2009b, MNRAS, 397, 232

Beirão, P., Santos, N. C., Israelian, G., \& Mayor, M. 2005, A\&A, 438, 251

Bensby, T., Feltzing, S., \& Lundström, I. 2003, A\&A, 410, 527

Bensby, T., Feltzing, S., Lundström, I., \& Ilyin, I. 2005, A\&A, 433, 185

Bergemann, M., Lind, K., Collet, R., \& Asplund, M. 2011, J. Phys. Conf. Ser., 328, 012002

Boss, A. P. 1997, Science, 276, 1836

Boss, A. P. 2002, ApJ, 567, L149

Boss, A. P. 2006, ApJ, 643, 501

Bouchy, F., \& Sophie Team. 2006, in Tenth Anniversary of 51 Peg-b: Status of and prospects for hot Jupiter studies, eds. L. Arnold, F. Bouchy, \& C. Moutou (Paris: Frontier Group), 319

Bressan, A., Marigo, P., Girardi, L., et al. 2012, MNRAS, 427, 127

Burgasser, A. J. 2011, in Molecules in the Atmospheres of Extrasolar Planets, eds. J. P. Beaulieu, S. Dieters, \& G. Tinetti, ASP Conf. Ser., 450, 113

Burrows, A., Marley, M., Hubbard, W. B., et al. 1997, ApJ, 491, 856

Burrows, A., Hubbard, W. B., Lunine, J. I., \& Liebert, J. 2001, Rev. Mod. Phys. 73, 719

Campbell, B., Walker, G. A. H., \& Yang, S. 1988, ApJ, 331, 902

Casagrande, L., Ramírez, I., Meléndez, J., Bessell, M., \& Asplund, M. 2010, A\&A, 512, A54

Cassan, A., Kubas, D., Beaulieu, J.-P., et al. 2012, Nature, 481, 167

Chabrier, G., \& Baraffe, I. 2000, ARA\&A, 38, 337

Chabrier, G., Johansen, A., Janson, M., \& Rafikov, R. 2014, Protostars and Planets VI, 619

Cosentino, R., Lovis, C., Pepe, F., et al. 2012, in SPIE Conf. Ser., 8446,

da Silva, L., Girardi, L., Pasquini, L., et al. 2006, A\&A, 458, 609

Dekker, H., D’Odorico, S., Kaufer, A., Delabre, B., \& Kotzlowski, H. 2000, in Optical and IR Telescope Instrumentation and Detectors, eds. M. Iye, \& A. F Moorwood, Proc. SPIE, 4008, 534

Delgado Mena, E., Israelian, G., González Hernández, J. I., et al. 2010, ApJ, 725 2349

Fischer, D. A., \& Valenti, J. 2005, ApJ, 622, 1102

Frandsen, S., \& Lindberg, B. 1999, in Astrophysics with the NOT, Proc. of Conf.

Turku, Finland, eds. H. Karttunen, \& V. Piirola, 71

Ghezzi, L., Cunha, K., Schuler, S. C., \& Smith, V. V. 2010, ApJ, 725, 721

Gonzalez, G. 1997, MNRAS, 285, 403

Grether, D., \& Lineweaver, C. H. 2006, ApJ, 640, 1051

Hekker, S., \& Meléndez, J. 2007, A\&A, 475, 1003

Høg, E., Fabricius, C., Makarov, V. V., et al. 2000, A\&A, 355, L27

Howard, A. W., Sanchis-Ojeda, R., Marcy, G. W., et al. 2013, Nature, 503, 381

Jofré, E., Petrucci, R., Saffe, C., et al. 2015, A\&A, 574, A50

Kaufer, A., Stahl, O., Tubbesing, S., et al. 1999, The Messenger, 95, 8
Kharchenko, N. V., Scholz, R.-D., Piskunov, A. E., Röser, S., \& Schilbach, E. 2007, Astron. Nachr., 328, 889

Kurucz, R. 1993, ATLAS9 Stellar Atmosphere Programs and $2 \mathrm{~km} \mathrm{~s}^{-1}$ grid. Kurucz CD-ROM No. 13 (Cambridge, Mass.: Smithsonian Astrophysical Observatory)

Luhman, K. L. 2012, ARA\&A, 50, 65

Luhman, K. L., Joergens, V., Lada, C., et al. 2007, Protostars and Planets V, 443

Ma, B., \& Ge, J. 2014, MNRAS, 439, 2781

Maldonado, J., \& Villaver, E. 2016, A\&A, 588, A98

Maldonado, J., Martínez-Arnáiz, R. M., Eiroa, C., Montes, D., \& Montesinos, B. 2010, A\&A, 521, A12

Maldonado, J., Villaver, E., \& Eiroa, C. 2013, A\&A, 554, A84

Maldonado, J., Eiroa, C., Villaver, E., Montesinos, B., \& Mora, A. 2015, A\&A 579, A20

Marcy, G. W., \& Butler, R. P. 2000, PASP, 112, 137

Mashonkina, L., Gehren, T., Shi, J.-R., Korn, A. J., \& Grupp, F. 2011, A\&A, 528, A87

Mata Sánchez, D., González Hernández, J. I., Israelian, G., et al. 2014, A\&A, 566, A83

Mayor, M., Pepe, F., Queloz, D., et al. 2003, The Messenger, 114, 20

Mayor, M., Marmier, M., Lovis, C., et al. 2011, A\&A, submitted [arXiv: 1109.2497]

Mendigutía, I., Calvet, N., Montesinos, B., et al. 2011, A\&A, 535, A99

Mendigutía, I., Mora, A., Montesinos, B., et al. 2012, A\&A, 543, A59

Montes, D., López-Santiago, J., Gálvez, M. C., et al. 2001, MNRAS, 328, 45

Mordasini, C., Alibert, Y., Benz, W., Klahr, H., \& Henning, T. 2012, A\&A, 541 , A97

Mortier, A., Santos, N. C., Sousa, S. G., et al. 2013, A\&A, 557, A70

Murdoch, K. A., Hearnshaw, J. B., \& Clark, M. 1993, ApJ, 413, 349

Muzerolle, J., Hillenbrand, L., Calvet, N., Briceño, C., \& Hartmann, L. 2003 ApJ, 592, 266

Natta, A., Testi, L., Muzerolle, J., et al. 2004, A\&A, 424, 603

Neves, V., Santos, N. C., Sousa, S. G., Correia, A. C. M., \& Israelian, G. 2009 A\&A, 497, 563

Niedzielski, A., Nowak, G., Adamów, M., \& Wolszczan, A. 2009, ApJ, 707, 768

Nordström, B., Mayor, M., Andersen, J., et al. 2004, A\&A, 418, 989

Pasquini, L., Döllinger, M. P., Weiss, A., et al. 2007, A\&A, 473, 979

Peacock, J. A. 1983, MNRAS, 202, 615

Perryman, M. A. C., \& ESA 1997, The Hipparcos and Tycho catalogues, Astrometric and photometric star catalogues derived from the ESA Hipparcos Space Astrometry Mission, ESA SP, 1200

Pollack, J. B., Hubickyj, O., Bodenheimer, P., et al. 1996, Icarus, 124, 62

Raskin, G., van Winckel, H., Hensberge, H., et al. 2011, A\&A, 526, A69

Reffert, S., Bergmann, C., Quirrenbach, A., Trifonov, T., \& Künstler, A. 2015 A\&A, 574, A116

Rice, W. K. M., \& Armitage, P. J. 2003, ApJ, 598, L55

Rice, W. K. M., Armitage, P. J., Bate, M. R., \& Bonnell, I. A. 2003a, MNRAS, 339, 1025

Rice, W. K. M., Armitage, P. J., Bonnell, I. A., et al. 2003b, MNRAS, 346, L36

Sadakane, K., Ohnishi, T., Ohkubo, M., \& Takeda, Y. 2005, PASJ, 57, 127

Sahlmann, J., Ségransan, D., Queloz, D., et al. 2011, A\&A, 525, A95

Santos, N. C., Israelian, G., \& Mayor, M. 2004, A\&A, 415, 1153

Santos, N. C., Sousa, S. G., Mortier, A., et al. 2013, A\&A, 556, A150

Schuler, S. C., Kim, J. H., Tinker, Jr., M. C., et al. 2005, ApJ, 632, L131

Sneden, C. A. 1973, Ph.D. Thesis, The University of Texas at Austin, USA

Sousa, S. G., Santos, N. C., Mayor, M., et al. 2008, A\&A, 487, 373

Sousa, S. G., Santos, N. C., Adibekyan, V., Delgado-Mena, E., \& Israelian, G. 2015, A\&A, 577, A67

Spiegel, D. S., Burrows, A., \& Milsom, J. A. 2011, ApJ, 727, 57

Stamatellos, D., \& Whitworth, A. P. 2009, MNRAS, 392, 413

Stevens, D. J., \& Gaudi, B. S. 2013, PASP, 125, 933

Takeda, Y. 2007, PASJ, 59, 335

Takeda, Y., Ohkubo, M., \& Sadakane, K. 2002a, PASJ, 54, 451

Takeda, Y., Sato, B., Kambe, E., Sadakane, K., \& Ohkubo, M. 2002b, PASJ, 54, 1041

Takeda, Y., Ohkubo, M., Sato, B., Kambe, E., \& Sadakane, K. 2005, PASJ, 57, 27

Takeda, Y., Sato, B., \& Murata, D. 2008, PASJ, 60, 781

Valenti, J. A., \& Fischer, D. A. 2005, ApJS, 159, 141

van Leeuwen, F. 2007, Hipparcos, the New Reduction of the Raw Data, Astrophys. Space Sci. Lib., 350

Wenger, M., Ochsenbein, F., Egret, D., et al. 2000, A\&AS, 143, 9

Whitworth, A., Bate, M. R., Nordlund, Å., Reipurth, B., \& Zinnecker, H. 2007, Protostars and Planets V, 459

Wilson, P. A., Hébrard, G., Santos, N. C., et al. 2016, A\&A, 588, A144

Zieliński, P., Niedzielski, A., Wolszczan, A., Adamów, M., \& Nowak, G. 2012, A\&A, 547, A91 
Appendix A: Additional tables

Table A.1. Spectroscopic parameters with uncertainties for the stars measured in this work.

\begin{tabular}{|c|c|c|c|c|c|c|c|c|c|c|c|}
\hline Star & $\begin{array}{c}M_{\mathrm{C}} \sin i^{\star} \\
\left(M_{\mathrm{Jup}}\right) \\
(2)\end{array}$ & $\begin{array}{l}T_{\text {eff }} \\
(\mathrm{K}) \\
(3)\end{array}$ & $\begin{array}{c}\log g \\
\left(\mathrm{~cm} \mathrm{~s}^{-2}\right) \\
(4)\end{array}$ & $\begin{array}{c}\xi_{t} \\
\left(\mathrm{~km} \mathrm{~s}^{-1}\right) \\
(5)\end{array}$ & $\begin{array}{c}{[\mathrm{Fe} / \mathrm{H}]} \\
(\mathrm{dex}) \\
(6)\end{array}$ & Sp..$^{\dagger}$ & $\begin{array}{l}\text { Age } \\
(\mathrm{Gyr}) \\
(8)\end{array}$ & $\begin{array}{c}M_{\star} \\
\left(M_{\odot}\right) \\
(9)\end{array}$ & $\begin{array}{l}R_{\star} \\
\left(R_{\odot}\right) \\
(10)\end{array}$ & $\mathrm{LC}^{\sharp}$ & $\operatorname{Kin}^{+}$ \\
\hline HD 4747 & $46.1 \pm 2.3 \mathrm{a}$ & $5373 \pm 20$ & $4.66 \pm 0.05$ & $0.84 \pm 0.19$ & $-0.18 \pm 0.02$ & 5 & $1.53 \pm 1.39$ & $0.85 \pm 0.01$ & $0.76 \pm 0.01$ & 5 & $\mathrm{D}$ \\
\hline HD 5388 & $69.2 \pm 19.9 \mathrm{a}, \mathrm{tm}$ & $6116 \pm 18$ & $3.75 \pm 0.03$ & $1.55 \pm 0.08$ & $-0.42 \pm 0.01$ & 2 & $5.29 \pm 0.34$ & $1.10 \pm 0.02$ & $1.93 \pm 0.07$ & 4 & $\mathrm{D}$ \\
\hline HIP 5158 & $15.04 \pm 10.55 \mathrm{a}$ & $4750 \pm 35$ & $4.71 \pm 0.08$ & $0.35 \pm 0.39$ & $0.36 \pm 0.04$ & 2 & $3.04 \pm 3.17$ & $0.80 \pm 0.02$ & $0.73 \pm 0.02$ & 5 & $\mathrm{D}$ \\
\hline HD 10697 & $38 \pm 13 \mathrm{a}, \mathrm{tm}$ & $5634 \pm 18$ & $4.03 \pm 0.04$ & $1.14 \pm 0.16$ & $0.12 \pm 0.03$ & 4 & $7.40 \pm 0.22$ & $1.11 \pm 0.02$ & $1.74 \pm 0.03$ & 4 & $\mathrm{D}$ \\
\hline HD 13189 & $20 \mathrm{a}$ & $4168 \pm 25$ & $1.63 \pm 0.10$ & $1.57 \pm 0.15$ & $-0.37 \pm 0.05$ & 4 & $4.50 \pm 2.88$ & $1.23 \pm 0.25$ & $33.69 \pm 5.93$ & 3 & $\mathrm{D}$ \\
\hline HD 13507 & $67_{-9}^{+8} \mathrm{~b}$ & $5726 \pm 18$ & $4.61 \pm 0.04$ & $0.94 \pm 0.10$ & $-0.03 \pm 0.02$ & 1 & $1.57 \pm 1.19$ & $0.99 \pm 0.02$ & $0.91 \pm 0.01$ & 5 & $\mathrm{D}$ \\
\hline HD 14348 & $48.9 \pm 1.6 \mathrm{~b}$ & $6095 \pm 23$ & $4.09 \pm 0.05$ & $1.26 \pm 0.09$ & $0.17 \pm 0.02$ & 1 & $3.19 \pm 0.10$ & $1.31 \pm 0.02$ & $1.63 \pm 0.05$ & 4 & $\mathrm{D}$ \\
\hline HD 14651 & $47 \pm 3.4 \mathrm{a}$ & $5490 \pm 8$ & $4.57 \pm 0.02$ & $0.83 \pm 0.06$ & $-0.03 \pm 0.01$ & 2 & $8.36 \pm 2$ & $0.89 \pm$ & $0.91 \pm$ & 5 & TR \\
\hline HD 16760 & $13.13 \pm 0.56 \mathrm{a}$ & $5614 \pm 15$ & $4.61 \pm 0.04$ & $0.69 \pm 0.09$ & $-0.02 \pm 0.01$ & 1 & $2.78 \pm 2.72$ & $0.95 \pm 0.02$ & $0.91 \pm 0.04$ & 5 & $\mathrm{D}$ \\
\hline HD 22781 & $13.65 \pm 0.97 \mathrm{a}$ & $5175 \pm 15$ & $4.57 \pm 0.04$ & $0.15 \pm 0.35$ & $-0.35 \pm 0.02$ & 1 & $4.14 \pm 3.63$ & $0.75 \pm 0.02$ & $0.70 \pm 0.02$ & 5 & $\mathrm{D}$ \\
\hline HD 283668 & $53 \pm 4 b$ & $4860 \pm 25$ & $4.65 \pm 0.06$ & $0.03 \pm 0.25$ & $-0.78 \pm 0.01$ & 1 & $5.90 \pm 4.22$ & $0.62 \pm$ & $0.57=$ & 5 & TR \\
\hline HIP 21832 & $40.9 \pm 26.2 \mathrm{a}, \mathrm{tm}$ & $5570 \pm 15$ & $4.37 \pm 0.04$ & $0.87 \pm 0.11$ & $-0.61 \pm 0.01$ & 1 & $11.33 \pm 0.30$ & $0.74 \pm 0.00$ & $0.76 \pm$ & 5 & TR \\
\hline HD 30246 & $55.1_{-8.2}^{+20.3} \mathrm{a}$ & $5795 \pm 15$ & $4.58 \pm 0.03$ & $1.10 \pm 0.07$ & $0.12 \pm 0.01$ & 2 & $0.95 \pm 0.81$ & $1.07 \pm 0.01$ & $0.98 \pm 0.03$ & 5 & $\mathrm{D}$ \\
\hline HD 39091 & $10.27 \pm 0.84 b$ & $5941 \pm 10$ & $4.33 \pm 0.02$ & $1.15 \pm 0.04$ & $0.03 \pm 0.01$ & 2 & $4.96 \pm 0.26$ & $1.07 \pm 0.01$ & $1.15 \pm 0.00$ & 5 & $\mathrm{D}$ \\
\hline HD 38529 & $13.99 \pm 0.59 \mathrm{a}$ & $5578 \pm 43$ & $3.78 \pm 0.12$ & $1.18 \pm 0.14$ & $0.31 \pm 0.04$ & 6 & $3.88 \pm 0.11$ & $1.38 \pm 0.02$ & $2.47 \pm 0.07$ & 4 & $\mathrm{D}$ \\
\hline HD 39392 & $13.2 \pm 0.8 b$ & $5824 \pm 15$ & $3.71 \pm 0.03$ & $1.14 \pm 0.06$ & $-0.54 \pm 0.01$ & 1 & $9.06 \pm 1.40$ & $0.94 \pm 0.04$ & $1.72 \pm 0.14$ & 4 & TR \\
\hline NGC 2423-3 & $10.64 \pm 0.93 \mathrm{a}$ & $4630 \pm 20$ & $2.44 \pm 0.08$ & $1.35 \pm 0.09$ & $0.00 \pm 0.04$ & 2 & & & & 3 & $\mathrm{D}$ \\
\hline HD 65430 & $67.8 \mathrm{a}$ & $5188 \pm 18$ & $4.68 \pm 0.05$ & $0.50 \pm 0.28$ & $-0.11 \pm 0.02$ & 1 & $10.13 \pm 1.51$ & $0.80 \pm 0.01$ & $0.79 \pm 0.01$ & 5 & $\mathrm{D}$ \\
\hline HD 72946 & $60.4 \pm 2.2 \mathrm{~b}$ & $6240 \pm 20$ & $4.29 \pm 0.05$ & $1.35 \pm 0.12$ & $0.03 \pm 0.02$ & 3 & & & & 5 & $\mathrm{D}$ \\
\hline HAT-P-13 & $14.28 \pm 0.28 \mathrm{a}$ & $5853 \pm 28$ & $4.41 \pm 0.06$ & $0.91 \pm 0.15$ & $0.48 \pm 0.03$ & 1 & 3. & 1.22 & 08 & 4 & $\mathrm{D}$ \\
\hline HD 77065 & $41 \pm$ & 503 & $4.74 \pm 0$ & $0.07 \pm$ & $-0.42 \pm$ & 1 & 7. & 1 & & 5 & TR \\
\hline $\mathrm{BD}+261888$ & $26 \pm 2 b$ & $4798 \pm 40$ & $4.54 \pm 0.09$ & $0.56 \pm 0.35$ & $0.04 \pm 0.04$ & 1 & $2.90 \pm 3$ & $0.77 \pm 0$ & 02 & 5 & $\mathrm{D}$ \\
\hline $\mathrm{BD}+202457$ & 22.7 & $4249 \pm 18$ & $1.62 \pm 0$ & $1.58 \pm$ & $-0.77 \pm$ & 4 & $4.54 \pm 4.06$ & $0.40=$ & 0.34 & 3 & TD \\
\hline $\mathrm{BD}+$ & & & & & & & & & & & \\
\hline HD 8 & $a$ & $5894 \pm 35$ & $4.23 \pm 0.08$ & $1.18 \pm 0.22$ & $-0.53 \pm 0.03$ & 3 & $11.13 \pm 0.49$ & $0.84 \pm 0.01$ & $1.02 \pm 0.02$ & 5 & TD \\
\hline HD 92320 & $59.4 \pm 4.1 \mathrm{a}$ & $5706 \pm 10$ & $4.64 \pm 0$ & $0.64 \pm$ & $-0.06=$ & 1 & 70 & 01 & & 5 & $\mathrm{D}$ \\
\hline $11 \mathrm{Com}$ & $5 \mathrm{a}$ & 8 & $2.52 \pm 0.03$ & $1.38 \pm$ & $-0.31 \pm 0.02$ & 8 & $1.17 \pm 0.28$ & $2.02 \pm 0.11$ & $14.88 \pm 0.36$ & 3 & TR \\
\hline NGC 4349-127 & $20 \pm 1.73 \mathrm{a}$ & $4439 \pm 28$ & $1.85 \pm 0.11$ & $1.58 \pm 0.13$ & $-0.18 \pm 0.05$ & 2 & & & & 3 & TD \\
\hline HD 114762 & $10.99 \pm 0.09 \mathrm{a}$ & $5851 \pm 28$ & $4.15 \pm 0.05$ & $1.25 \pm 0.18$ & $-0.74 \pm 0.02$ & 3 & $11.48 \pm 0.01$ & $0.85 \pm 0.00$ & $1.52 \pm 0.04$ & 5 & TD \\
\hline HD 122562 & $24 \pm 2 b$ & $4983 \pm 28$ & $3.86 \pm 0.09$ & $0.78 \pm 0.16$ & $0.31 \pm 0.04$ & 1 & $7.97 \pm 0.97$ & $1.12 \pm$ & $2.06 \pm 0.09$ & 4 & $\mathrm{D}$ \\
\hline HD 132032 & $70 \pm 4 b$ & $5954 \pm 13$ & $4.41 \pm 0.03$ & $0.98 \pm 0.08$ & $0.09 \pm 0.01$ & 1 & $2.87 \pm 1.54$ & $1.10 \pm 0.01$ & $1.12 \pm 0.06$ & 5 & $\mathrm{D}$ \\
\hline HD 131664 & $23_{-5.0}^{+26.0} \mathrm{a}, \mathrm{tm}$ & $5882 \pm 8$ & $4.49 \pm 0.02$ & $1.09 \pm 0.06$ & $0.30 \pm 0.01$ & 2 & $2.12 \pm 1.06$ & $1.15 \pm 0.01$ & $1.14 \pm 0.05$ & 5 & $\mathrm{D}$ \\
\hline HD 134113 & $47_{-3}^{+2} \mathrm{~b}$ & $5561 \pm 23$ & $3.76 \pm 0.05$ & $1.03 \pm 0.10$ & $-0.92 \pm 0.02$ & 1 & $10.98 \pm 0.66$ & $0.85 \pm 0.02$ & $2.01 \pm 0.07$ & 4 & TD \\
\hline HD 136118 & $2 \pm 0.47 \mathrm{a}$ & $6163 \pm 98$ & $3.81 \pm 0.19$ & $1.32 \pm 0.23$ & $-0.17 \pm 0.06$ & 1 & $4.94 \pm 1.05$ & $1.12 \pm 0.04$ & $1.48 \pm 0.06$ & 4 & $\mathrm{D}$ \\
\hline HD 137759 & $12.7 \pm 1.08 \mathrm{a}$ & $4647 \pm 38$ & & & & 6 & & & & 3 & $\mathrm{D}$ \\
\hline $\mathrm{HD}$ & & $5999 \pm 43$ & $4.13 \pm$ & & & 1 & & & & 4 & $\mathrm{D}$ \\
\hline HD 1 & $43.2 \mathrm{a}$ & $6071 \pm 115$ & $4.80 \pm 0.24$ & $1.68 \pm 0.50$ & $-0.08 \pm 0.07$ & 1 & $1.62 \pm 1.58$ & $1.02 \pm$ & $0.96=$ & 5 & $\mathrm{D}$ \\
\hline HD 1 & $10.57 \pm 0.29 b$ & $6051 \pm 13$ & $4.00 \pm 0.03$ & $1.39 \pm 0.05$ & $0.13 \pm 0.01$ & 2 & $3.38 \pm 0.41$ & $1.36 \pm 0.04$ & $1.91 \pm 0.05$ & 4 & $\mathrm{D}$ \\
\hline HD 160508 & $48 \pm 3 b$ & $6045 \pm 20$ & $3.77 \pm 0.04$ & $1.33 \pm 0.08$ & $-0.16 \pm 0.02$ & 1 & $5.55 \pm 0.57$ & $1.14 \pm 0.04$ & $1.76 \pm 0.13$ & 4 & $\mathrm{D}$ \\
\hline HD 162020 & $14.4 \pm 0.04 \mathrm{a}$ & $4801 \pm 30$ & $4.60 \pm 0.08$ & $0.62 \pm 0.34$ & $0.00 \pm 0.04$ & 2 & $3.32 \pm 3.35$ & $0.76 \pm 0.02$ & $0.70 \pm 0.02$ & 5 & $\mathrm{D}$ \\
\hline HD 167665 & $50.6 \pm 1.7 \mathrm{a}$ & $6080 \pm 15$ & $4.13 \pm 0.03$ & $1.29 \pm 0.08$ & $-0.21 \pm 0.01$ & 5 & $6.72 \pm 0.23$ & $1.03 \pm 0.01$ & $1.32 \pm 0.02$ & 5 & $\mathrm{D}$ \\
\hline HD 168443 & $34.3 \pm 9 \mathrm{a}, \mathrm{tm}$ & $5544 \pm 5$ & $4.11 \pm 0.02$ & $1.04 \pm 0.04$ & $0.01 \pm 0.01$ & 2 & $10.70 \pm 0.46$ & $1.00 \pm 0.01$ & $1.54 \pm 0.04$ & 4 & $\mathrm{D}$ \\
\hline HD 174457 & $65.8 \mathrm{~b}$ & $5825 \pm 20$ & $4.08 \pm 0.05$ & $1.22 \pm 0.12$ & $-0.26 \pm 0.02$ & 3 & $9.80 \pm 0.55$ & $0.96 \pm 0.02$ & $1.48 \pm 0.07$ & 4 & $\mathrm{D}$ \\
\hline HD 175679 & $37.3 \pm 2.8 \mathrm{a}$ & $5028 \pm 33$ & $2.57 \pm 0.10$ & $1.43 \pm 0.17$ & $-0.01 \pm 0.05$ & 5 & $0.66 \pm 0.11$ & $2.53 \pm 0.12$ & $11.79 \pm 0.80$ & 3 & $\mathrm{D}$ \\
\hline HD 180314 & $22 \mathrm{a}$ & $4983 \pm 53$ & $3.17 \pm 0.17$ & $1.27 \pm 0.21$ & $0.24 \pm 0.07$ & 4 & $1.14 \pm 0.24$ & $2.13 \pm 0.13$ & $8.88 \pm 0.47$ & 3 & TR \\
\hline KOI-415 & $62.14 \pm 2.69 \mathrm{~b}$ & $5513 \pm 78$ & $4.36 \pm 0.20$ & $0.80 \pm 0.31$ & $0.15 \pm 0.07$ & 7 & & & & 4 & \\
\hline HD 190228 & $49.4 \pm 14.8 \mathrm{a}, \mathrm{tm}$ & $5241 \pm 20$ & $3.66 \pm 0.05$ & $0.96 \pm 0.09$ & $-0.33 \pm 0.02$ & 3 & 5.7 & 1.1 & & 4 & $\mathrm{D}$ \\
\hline HR 7672 & & & & & $-0.01 \pm$ & 3 & & & & 5 & $\mathrm{D}$ \\
\hline HD 191760 & $38.17 \pm 1.0$ & & & & & 2 & & & & 4 & $\mathrm{D}$ \\
\hline HD 202206 & $17.5 \mathrm{a}$ & $5754 \pm 8$ & $4.56 \pm 0.02$ & $1.03 \pm 0.05$ & $0.29 \pm 0.01$ & 2 & $1.02 \pm 0.83$ & $1.10 \pm 0.01$ & $1.02 \pm 0.03$ & 5 & $\mathrm{D}$ \\
\hline HD 209262 & $32.3_{-1.5}^{+1.6} \mathrm{~b}$ & $5753 \pm 8$ & $4.38 \pm 0.02$ & $1.02 \pm 0.04$ & $0.06 \pm 0.01$ & 2 & $7.48 \pm 0.75$ & $1.00 \pm 0.01$ & $1.13 \pm 0.05$ & 5 & $\mathrm{D}$ \\
\hline $\mathrm{BD}+244697$ & $53 \pm 3 b$ & $4937 \pm 25$ & $4.74 \pm 0.07$ & $0.12 \pm 0.46$ & $-0.16 \pm 0.03$ & 1 & $5.207 \pm 4.15$ & $0.754 \pm 0.016$ & $0.705 \pm 0.017$ & 5 & TR \\
\hline HD 217786 & $13 \pm 0.8 \mathrm{a}$ & $5882 \pm 8$ & $4.13 \pm 0.02$ & $1.18 \pm 0.05$ & $-0.19 \pm 0.01$ & 2 & $9.40 \pm 0.22$ & $0.96 \pm 0.01$ & $1.32 \pm 0.06$ & 4 & $\mathrm{D}$ \\
\hline HD 219077 & $10.39 \pm 0.09 \mathrm{~b}$ & $5284 \pm 5$ & $3.91 \pm 0.02$ & $0.94 \pm 0.04$ & $-0.18 \pm 0.01$ & 2 & $8.55 \pm 0.25$ & $1.03 \pm 0.01$ & $1.99 \pm 0.02$ & 4 & TR \\
\hline
\end{tabular}

Notes. ${ }^{\star}{ }^{\star}$ (a) Ma \& Ge (2014, and references therein), (b) Wilson et al. (2016, and references therein), (tm) "true" mass. ( ${ }^{\dagger)}$ Spectrograph: (1) SOPHIE; (2) ESO/HARPS; (3) ELODIE; (4) NOT/FIES; (5) ESO/FEROS; (6) Mercator/HERMES; (7) TNG/HARPS-N; (8) ESO/UVES. (\$) 5: main-sequence; 4: subgiant; 3: giant. ${ }^{(\ddagger)}$ D: thin disc, TD: thick disc, TR: transition. 
Table A.2. Derived abundances $[\mathrm{X} / \mathrm{H}]$.

\begin{tabular}{|c|c|c|c|c|c|c|c|c|c|c|c|c|c|c|c|c|c|c|c|}
\hline Star & I & $\mathrm{O}_{\mathrm{I}}$ & $\mathrm{a} I$ & Mg I & $\mathrm{Al} \mathrm{I}$ & I & I & $\mathrm{Ca} \mathrm{I}$ & $c I$ & Sc II & I & II & V I & $\mathrm{r} I$ & Cr II & Mn I & o I & $\mathrm{Ni} \mathrm{I}$ & $\mathrm{Zn} \mathrm{I}$ \\
\hline \multirow[t]{2}{*}{ HD 4747} & & & & & 20 & -0.19 & .07 & -0.22 & 26 & & & & 6 & 20 & .18 & 0 & 1 & 27 & 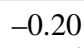 \\
\hline & & & 0.04 & & .04 & .02 & 0.05 & & & & & & & & & & & & \\
\hline \multirow[t]{2}{*}{ HD 5388} & 31 & -0.42 & -0.30 & 37 & -0.46 & -0.32 & & -0.31 & & -0.51 & -0.38 & & & & 41 & 50 & 50 & - & 53 \\
\hline & 03 & 0.04 & 0.06 & 0.07 & 0.03 & 0.02 & & 0.04 & & & 0.03 & & 0.06 & & 0.06 & & & 0.02 & 0.05 \\
\hline \multirow[t]{2}{*}{ HIP 5158} & 09 & -0.05 & 0.36 & 0.15 & 0.41 & 0.34 & & 0.09 & & & 0.28 & 0.32 & 0.64 & 0.33 & 0.44 & 52 & 0.42 & 0.37 & \\
\hline & 07 & 0.07 & 0.12 & 0.07 & 0.06 & 0.04 & & 0.13 & & & 0.10 & 0.10 & 0.07 & 0.07 & 0.12 & 0.13 & 0.06 & 0.06 & \\
\hline \multirow[t]{2}{*}{ HD 10697} & 0.07 & 01 & 0.13 & 0.23 & 0.18 & 0.12 & 0.07 & 0.11 & 0.02 & & 0.09 & & 0.07 & 0.10 & 0.14 & 0.22 & 0.08 & 0.10 & 0.07 \\
\hline & & .06 & 0.04 & & 0.04 & 0.02 & 0.15 & 0.06 & 0.06 & 0.06 & 0.05 & & 0.05 & 0.05 & 0.08 & & 0.06 & 0.04 & 0.08 \\
\hline \multirow[t]{2}{*}{ HD 13189} & & & -0.19 & -0.22 & -0.09 & -0.17 & & -0.28 & & -0.31 & -0.16 & -0.15 & 0.04 & -0.34 & -0.32 & -0.01 & -0.12 & -0.29 & \\
\hline & & & & & 0.06 & 0.06 & & 0.09 & & & 0.11 & & 0.17 & 0.09 & 0.14 & 13 & 0.10 & 0.07 & \\
\hline \multirow[t]{2}{*}{ HD 13507} & & & -0.17 & & -0.14 & & .01 & -0.04 & -0.07 & -0.15 & -0.05 & & -0.05 & -0.02 & 02 & & -0.14 & -0.12 & -0.28 \\
\hline & & & & & & & & & & & & & & & & & & 0.03 & 0.04 \\
\hline HD 14348 & & & & & & & & & & & & & & & & & 6 & 17 & 0.07 \\
\hline & & & & & & & & & & & & & & & & & & & 0.06 \\
\hline HD 1 & & & & & 0 & & 0.02 & -0.08 & -0.07 & 6 & 02 & & .02 & 0.02 & & & 3 & -0.05 & -0.06 \\
\hline & & 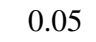 & & & 0.03 & & 0.05 & 0.06 & 0.05 & & & & & & & & & 4 & 0.08 \\
\hline 760 & & & -0 . & 4 & -0.08 & -0.08 & -0.19 & -0.06 & -0.14 & -0 . & -0.06 & -0 & 0.07 & & & & 0.17 & -0.09 & 0.05 \\
\hline & & & 0.04 & 0.0 & 0.04 & 0.02 & 0.05 & 0.06 & 0.03 & & 0.04 & & 0.04 & 0.04 & & & 0.07 & 0.03 & 0.14 \\
\hline 81 & -0.05 & & -0.24 & -0.26 & 0.11 & -0.28 & & -0.21 & -0.07 & -0.12 & -0.05 & -0.15 & -0.06 & -0.27 & -0.25 & & -0.25 & -0.36 & -0.18 \\
\hline & 0 & & 010 & & 0.15 & 003 & & 008 & 0.10 & & 0.08 & & 0.07 & 0.06 & & & & 0.05 & 0.16 \\
\hline HD 283668 & 0 & & & & -0.40 & -0 . & & 3 & -0.44 & & & & 43 & & & & & & -0.68 \\
\hline & & & & & & & & & 0.07 & & & & & & & & & & 0.09 \\
\hline $\mathrm{F}$ & -0.48 & & -0 & & -0.44 & -0.46 & & -0.48 & & -0 & -0.43 & & -0.44 & & & & -0.54 & -0.62 & -0.61 \\
\hline & & & & & 0.04 & & & & & & & & & & & & & & 0.07 \\
\hline HD 30246 & & & & & 0. & & & & & & & & & & & & & & 0.02 \\
\hline & & & & & & & & & & & & & & & & & & & 11 \\
\hline HD? & & & & & & & & & & & & & & & & & & 2 & -0.04 \\
\hline & & & & & & & & & & & & & & & & & & & 0.07 \\
\hline HD & & & & & & & & & & & & & & & & & & & .22 \\
\hline & & 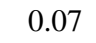 & & & & & & & & & & & & & & & & & 11 \\
\hline 92 & -0.38 & & 0 & -0. & -0.48 & -0.44 & -0.28 & -0.4 & & -0 & -0.52 & 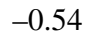 & -0.55 & -0.56 & & & 1 & & -0.60 \\
\hline & & & 0.11 & 0.0 & 0.04 & 0.02 & 0.04 & 0. & & & 0.04 & & 0.10 & 0.05 & & & & 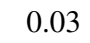 & 0.04 \\
\hline NGC 2 & -0.10 & .03 & 0.10 & 0.26 & 0.10 & 0.09 & & -0.04 & -0.01 & -0 . & 0.05 & -0 & 0.07 & 0.01 & & & & -0 & -0.43 \\
\hline & & & & & & & & & & & & & & & & & & & 0.08 \\
\hline F & -0.02 & -0.05 & -0.11 & -0.0 & 0.05 & -0 . & & 16 & -0.05 & & & & -0.01 & -0.13 & & & & & 0.26 \\
\hline & & & & & 0.04 & & & & & & & & & & & & & & .08 \\
\hline H & & & 0.07 & -0. & & 0.04 & -0.05 & 0.01 & & -0 . & & -0 & & & & & & -0 & -0.18 \\
\hline & & & & & & & 0.17 & & & & & & & & & & & & 0.06 \\
\hline HAT-P-13 & & & & & & & & & & & & & & & & & & & .29 \\
\hline & & & & & & & & & & & & & & & & & & & .04 \\
\hline 65 & & & & & & -0 . & & 1 & -0.23 & & & & & & & & & & -0.21 \\
\hline & & & & & & & & & & & & & & & & & & & 0.08 \\
\hline $\mathrm{BD}$ & & & & & & & & & & & & & & & & & & & .09 \\
\hline & & & & & & & & & & & & & & & & & & & 15 \\
\hline 7 & & -0.26 & -0.8 & -0 . & -0.66 & -0.5 & & 5 & -0.74 & -0 & -0 & -0 & -0 & -0 & -0 & & & -0 & -0.73 \\
\hline & & 0.07 & & & 0.05 & & & & 0.16 & & & & & & & & & & .10 \\
\hline & -0.62 & & -0.34 & -0.4 & & -0.3 & & -0.41 & & -0 & -0.42 & -0 & -0.60 & -0.55 & -0 & & & -0.55 & -0.49 \\
\hline & & & & & & & & & & & & & & & & & & 3 & 0.04 \\
\hline $\mathrm{F}$ & -0 & & -0 . & -0 . & & & & -0.09 & & & -0.09 & & -0.15 & -0.07 & -0.02 & & & -0 & -0.16 \\
\hline & & & & & & & & & & & & & & & & & & & 0.06 \\
\hline $11 \mathrm{Com}$ & -0.45 & -0.12 & -0.05 & -0.19 & - & -0.16 & & -0.20 & -0.43 & -0.25 & -0.23 & -0.35 & -0.25 & -0.31 & -0.24 & 27 & -0.24 & -0.30 & -0.37 \\
\hline & & & & & & & & & 0.21 & & & & & & & & 98 & 0.07 & 0.15 \\
\hline NGC 4 & -0.20 & -0.21 & & & -0.12 & & & -0.14 & & -0.23 & -0.19 & -0.07 & -0.06 & & & 3 & -0.13 & -0.20 & \\
\hline & & & & & & & & & & & & & & & & & 0.07 & & \\
\hline HD 114762 & -0.51 & & -0.56 & -0.53 & -0.45 & -0.55 & & -0.52 & & & -0.54 & & & & & & & -0.75 & -0.66 \\
\hline & & & & & & & & & & & & & & & & & & & 0.02 \\
\hline 10 & & -0.05 & & & & & & & & & & & & & & & & & 0.49 \\
\hline & & 0.08 & & & & & & & & & & & & & & & & & 0.20 \\
\hline & & & & & & & & & & & & & & & & & & & 0.09 \\
\hline 1 & & & & & & & & & & & & & & & & & & 09 & 0.08 \\
\hline & & & & & & & & & & & & & & & 0 . & & . & 0.02 & 0.11 \\
\hline & & & & & & & & & & & & & & & & & & 0.32 & \\
\hline & & 0.03 & & & 0.02 & & 0.03 & & 0.02 & & & & & & & 33 & 0.05 & 0.02 & \\
\hline HD 134113 & -0.5 & & -0.69 & -0.55 & -0.56 & -0.61 & & -0.5 & & -0.92 & -0.70 & -0.68 & -0.82 & -0.93 & -0.83 & -1.17 & -0.83 & -0.90 & -0.79 \\
\hline & 0.06 & & 0.08 & 0.09 & 0.04 & 0.02 & & 0.08 & & 0.08 & 0.06 & 0.08 & 0.10 & 0.07 & 0.09 & 0.11 & 0.05 & 0.04 & 0.07 \\
\hline
\end{tabular}


Table A.2. continued.

\begin{tabular}{|c|c|c|c|c|c|c|c|c|c|c|c|c|c|c|c|c|c|c|c|}
\hline Star & I & $\mathrm{O} \mathrm{I}$ & I & Ig I & Al I & Si I & S I & $I$ & Sc I & II & Ti I & Ti II & V I & Cr I & Cr II & Mn I & o I & $\mathrm{Ni} \mathrm{I}$ & Z \\
\hline \multirow[t]{2}{*}{ HD 136118} & & & & & & & & & & & & & & & & & & & \\
\hline & & & & & & 0.09 & 0.04 & 0.06 & & & & & & 0.09 & & & & & \\
\hline \multirow[t]{2}{*}{ HD 137759} & & & 0.41 & 0.36 & 0.40 & 0.37 & & 0 . & & & & & & 0.23 & & & & & \\
\hline & & & 0.09 & 0.09 & 0.05 & 0.06 & & 0.1 & & & 2 & 0 . & & & & & & & \\
\hline \multirow[t]{2}{*}{ HD 137510} & 0.28 & & 0.55 & 0.21 & & 0.35 & 0.20 & 0.2 & & & 02 & 0.26 & & 0.20 & & & & & \\
\hline & & & 0.03 & 0.09 & & 0.02 & 0.03 & 0. & & & 0 & 0 & & 0.02 & & & & .02 & \\
\hline \multirow{2}{*}{ HD 140913} & & & -0.13 & & 16 & -0.17 & -0.07 & & & & 0.02 & & U & & & & 05 & 0.11 & -0.47 \\
\hline & & & & & & & & & & & & & & & & & & 06 & 0.0 \\
\hline \multirow[t]{2}{*}{ HD 156846} & 0.09 & & 0.24 & 0.15 & & 0.16 & & & & & & & & & & & & & .0 \\
\hline & & & & & & & & & & & & & & & & & & & 0.0 \\
\hline \multirow[t]{2}{*}{ HD 160508} & & & & & & & & & & & & & & & & & & & -0.2 \\
\hline & & & & & & & & & & & & & & & & & & & \\
\hline \multirow[t]{2}{*}{ HD 162020} & & & & & & & & & & & & & & & & & & & -0.0 \\
\hline & & & & & & & & & & & & & & & & & & & \\
\hline \multirow[t]{2}{*}{ HD 167665} & -0.0 & & & & & -0 . & 21 & & & 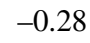 & & & & & & & & & -0 \\
\hline & & & & & & & & & & & & & & & & & & & 0 \\
\hline \multirow[t]{2}{*}{ HD 168443} & & 12 & & & & & & & & & & & & & & & & & .1 \\
\hline & & & & & & & 0.05 & & & & & & & & & & & & $0.2+2-3$ \\
\hline \multirow[t]{2}{*}{ HD 174457} & -0.06 & & -0.18 & -0.21 & -0.09 & -0.22 & -0.27 & -0.18 & & -0.29 & -0.22 & -0.12 & -0.16 & -0.17 & -0.18 & -0 & -0.23 & -0.31 & -0 \\
\hline & & & & & & & & & & & & & & & & & & & \\
\hline \multirow{2}{*}{ HD 175679} & 26 & .09 & & & & & & & 24 & -0.10 & & & & & & & 09 & & \\
\hline & & & & & & & & & & & & & & & & & & & \\
\hline \multirow[t]{2}{*}{ HD 180314} & & & & & & & & & & & & & & & & & & 26 & 0.3 \\
\hline & & & & & & & & & & & & & & & & & & & 0.1 \\
\hline \multirow[t]{2}{*}{ KOI-415 } & & & & & & & & & & & & & & & & & & & $32+2$ \\
\hline & & & & & & & & & & & & & & & & & & & 0.0 \\
\hline \multirow[t]{2}{*}{ HD 190228} & & & & & & & & & & & & & & & & & & & \\
\hline & & & & & & & & & & & & & & & & & & & \\
\hline \multirow[t]{2}{*}{ HR 7672} & & & & & & & & & & & & & & & & & & & -1 \\
\hline & & & & & & & & & & & & & & & & & & & \\
\hline \multirow[t]{2}{*}{ HD 191760} & & & & & & & & & & & & & & & & & & & 0.2 \\
\hline & & & & & & & & & & & & & & & & & & & 0.0 \\
\hline \multirow[t]{2}{*}{ HD 202206} & & & & & & & & & & & & & & & & & & & 0.1 \\
\hline & & & & & & & & & & & & & & & & & & & 0.1 \\
\hline \multirow[t]{2}{*}{ HD 209262} & & -0 . & & & & & & & & & & & & & & & & & 0. \\
\hline & & & & & & & 0.04 & & & & & & & & & & & 02 & 0.1 \\
\hline$D+244697$ & 0.42 & & -0.38 & -0.26 & -0.16 & -0.18 & & -0.23 & -0.14 & -0.30 & -0.10 & -0.15 & -0.07 & -0.17 & & -0.19 & -0.06 & -0.20 & -0.2 \\
\hline & & & & & & & & & & & & & & & & & & 06 & $0.0+3$ \\
\hline 786 & -0.08 & -0.25 & -0.12 & -0.11 & -0.16 & -0 . & & -0.15 & & -0.25 & -0.22 & -0.20 & -0.23 & -0.19 & -0.21 & -0.27 & -0.24 & -0.21 & -0.2 \\
\hline & & & & & & & & & & & & & & & & & & & 0. \\
\hline HD 2 & 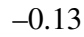 & -0.0 & -0 & & -0.03 & -0.10 & & -0.13 & & -0 & -0 & -0.07 & -0 & -0.18 & -0 & -0.19 & -0.20 & -0 & \\
\hline & & 0.07 & & 0.00 & 0.05 & 0.03 & 0.01 & 0.08 & 0.07 & 0.09 & 0.08 & 0.09 & 0.07 & 0.07 & 0.09 & 0.10 & 0.06 & 0.0 & \\
\hline
\end{tabular}

